Discrete Comput Geom 27:603-634 (2002)

DOI: $10.1007 / \mathrm{s} 00454-002-2776-6$

\title{
A Polytope Related to Empirical Distributions, Plane Trees, Parking Functions, and the Associahedron*
}

\author{
Richard P. Stanley ${ }^{1}$ and Jim Pitman ${ }^{2}$ \\ ${ }^{1}$ Department of Mathematics, Massachusetts Institute of Technology, \\ Cambridge, MA 02139, USA \\ rstan@math.mit.edu \\ 2 Department of Statistics, University of California, \\ 367 Evans Hall \#3860, Berkeley, CA 94720-3860, USA \\ pitman@stat.berkeley.edu
}

\begin{abstract}
The volume of the $n$-dimensional polytope

$$
\Pi_{n}(\boldsymbol{x}):=\left\{\boldsymbol{y} \in \mathbb{R}^{n}: y_{i} \geq 0 \text { and } y_{1}+\cdots+y_{i} \leq x_{1}+\cdots+x_{i} \text { for all } 1 \leq i \leq n\right\}
$$

for arbitrary $\boldsymbol{x}:=\left(x_{1}, \ldots, x_{n}\right)$ with $x_{i}>0$ for all $i$ defines a polynomial in variables $x_{i}$ which admits a number of interpretations, in terms of empirical distributions, plane partitions, and parking functions. We interpret the terms of this polynomial as the volumes of chambers in two different polytopal subdivisions of $\Pi_{n}(\boldsymbol{x})$. The first of these subdivisions generalizes to a class of polytopes called sections of order cones. In the second subdivision the chambers are indexed in a natural way by rooted binary trees with $n+1$ vertices, and the configuration of these chambers provides a representation of another polytope with many applications, the associahedron.
\end{abstract}

\section{Introduction}

The focal point of this paper is the $n$-dimensional polytope

$$
\Pi_{n}(\boldsymbol{x}):=\left\{\boldsymbol{y} \in \mathbb{R}^{n}: y_{i} \geq 0 \text { and } y_{1}+\cdots+y_{i} \leq x_{1}+\cdots+x_{i} \text { for all } 1 \leq i \leq n\right\}
$$

for arbitrary $\boldsymbol{x}:=\left(x_{1}, \ldots, x_{n}\right)$ with $x_{i}>0$ for all $i$. The $n$-dimensional volume

$$
V_{n}(\boldsymbol{x}):=\operatorname{Vol}\left(\Pi_{n}(\boldsymbol{x})\right)
$$

\footnotetext{
* The research of R. P. Stanley was supported in part by NSF Grants \#9743966 and \#9988459, and that by J. Pitman was supported in part by NSF Grant \#9703961.
} 

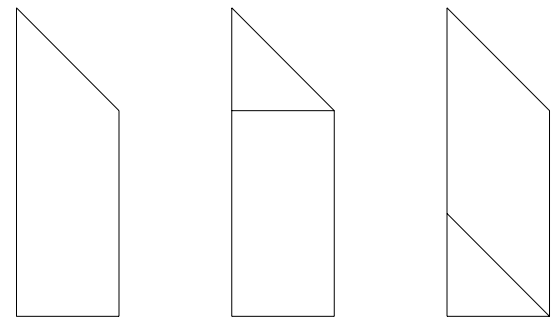

Fig. 1. $\Pi_{2}(\boldsymbol{x})$ and its two subdivisions.

is a homogeneous polynomial of degree $n$ in the variables $x_{1}, \ldots, x_{n}$, which we call the volume polynomial. This polynomial arises naturally in several different settings: in the calculation of probabilities derived from empirical distribution functions or the order statistics of $n$ independent random variables (see Section 2), and in the study of parking functions and plane partitions (see Section 5). See also [15] regarding similar connections between the theories of parking functions, empirical processes, and rooted trees.

Trivially, $V_{1}(\boldsymbol{x})=x_{1}$. The formula

$$
V_{2}(\boldsymbol{x})=x_{1} x_{2}+\frac{1}{2} x_{1}^{2}
$$

has two natural interpretations by a subdivision of $\Pi_{2}(\boldsymbol{x})$ into two pieces of areas $x_{1} x_{2}$ and $\frac{1}{2} x_{1}^{2}$, as shown in Fig. 1 for horizontal coordinate $x_{1}=1$ and vertical coordinate $x_{2}=2$.

The five terms of

$$
V_{3}(\boldsymbol{x})=x_{1} x_{2} x_{3}+\frac{1}{2} x_{1}^{2} x_{2}+\frac{1}{2} x_{1} x_{2}^{2}+\frac{1}{2} x_{1}^{2} x_{3}+\frac{1}{6} x_{1}^{3}
$$

can be interpreted in two ways as the volumes determined by two different subdivisions of $\Pi_{3}(\boldsymbol{x})$ into five chambers, as in the perspective diagrams of Fig. 2 where $x_{i}=i$ for $i=1,2,3$, the first coordinate points out of the page, the second to the right, and the third up, and the viewpoint is $(5,-2,4)$.

A central result of this paper is the general formula for the volume polynomial which we present in the following theorem. Section 2 offers a simple probabilistic proof of this theorem. We show in Section 4 how this argument can also be interpreted geometrically
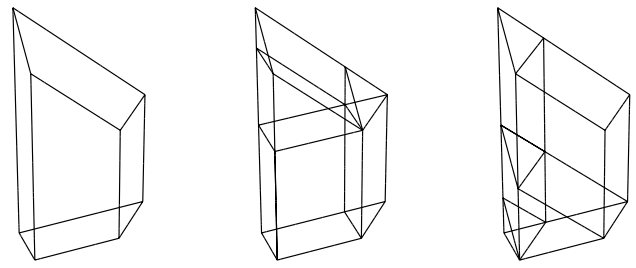

Fig. 2. $\Pi_{3}(\boldsymbol{x})$ and its two subdivisions 
by a subdivision of $\Pi_{n}(\boldsymbol{x})$ into a collection of $n$-dimensional chambers, with the volume of each chamber corresponding to a term of the volume polynomial. This generalizes the subdivisions of $\Pi_{2}$ and $\Pi_{3}$ shown in the right-hand panels of Figs. 1 and 2. Technically, by a subdivision of $\Pi_{n}(\boldsymbol{x})$ we mean a polytopal subdivision in the sense of Ziegler [39, p. 129 ], and we call the $n$-dimensional polytopes involved the chambers of the subdivision. The subdivision of $\Pi_{n}(\boldsymbol{x})$ described in Section 4 is a specialization of a result presented in Section 3 in the general context of "sections of order cones." Section 6 shows how the subdivisions shown in the left-hand panels of Figs. 1 and 2 can be generalized to arbitrary $n$. The chambers of this subdivision of $\Pi_{n}(\boldsymbol{x})$ are indexed in a natural way by rooted binary plane trees with $n+1$ leaf vertices, and the configuration of these chambers provides a representation of another interesting polytope with many applications, known as the associahedron.

Theorem 1. For each $n=1,2, \ldots$,

$$
V_{n}(\boldsymbol{x})=\sum_{\boldsymbol{k} \in K_{n}} \prod_{i=1}^{n} \frac{x_{i}^{k_{i}}}{k_{i} !}=\frac{1}{n !} \sum_{\boldsymbol{k} \in K_{n}}\left(\begin{array}{c}
n \\
k_{1}, \ldots, k_{n}
\end{array}\right) x_{1}^{k_{1}} \cdots x_{n}^{k_{n}},
$$

where

$$
K_{n}:=\left\{\boldsymbol{k} \in \mathbb{N}^{n}: \sum_{i=1}^{j} k_{i} \geq j \text { for all } 1 \leq j \leq n-1 \text { and } \sum_{i=1}^{n} k_{i}=n\right\}
$$

with $\mathbb{N}:=\{0,1,2, \ldots\}$.

In particular, the number of nonzero coefficients in $V_{n}$ is the number of elements of $K_{n}$, which is well known to be the $n$th Catalan number $C_{n}$ (see, e.g., Exercise 6.19(w) of [34] for a simple variant), the first few of which are $1,2,5,14,42,132, \ldots$ :

$$
\# K_{n}=C_{n}:=\frac{1}{n+1}\left(\begin{array}{c}
2 n \\
n
\end{array}\right) .
$$

Formula (2) should be compared with the following alternate formula, which as indicated in Section 2 can be read from a formula of Steck [36], [37] for the cumulative distribution function of the random vector of order statistics of $n$ independent random variables with uniform distribution on an interval:

$$
V_{n}(\boldsymbol{x})=\operatorname{det}\left[\frac{1(j-i+1 \geq 0)}{(j-i+1) !}\left(\sum_{h=1}^{i} x_{h}\right)^{j-i+1}\right]_{1 \leq i, j \leq n}
$$

where $\operatorname{det}\left[a_{i j}\right]_{1 \leq i, j \leq n}$ denotes the determinant of the $n \times n$ matrix with entries $a_{i j}$, and $1(\cdots)$ equals 1 if $\cdots$ and 0 else. See [23] for an elementary probabilistic proof of (5). This formula allows the expansion of $V_{n}(\boldsymbol{x})$ into monomial terms to be generated for arbitrary $n$ by just a few lines of Mathematica code.

Another formula of Steck [36], [37], with an elementary proof in [23], gives the number $\#(b, c)$ of $j \in \mathbb{Z}^{n}$ with $j_{1}<j_{2}<\cdots<j_{n}$ and $b_{i}<j_{i}<c_{i}$ for all $1 \leq i \leq n$ 
for arbitrary $b, c \in \mathbb{Z}^{n}$ with $b_{1} \leq b_{2} \leq \cdots<b_{n}$ and $c_{1} \leq c_{2} \leq \cdots<c_{n}$ :

$$
\#(b, c)=\operatorname{det}\left[1\left(j-i+1 \geq 0, c_{i}-b_{j}>1\right)\left(\begin{array}{c}
c_{i}-b_{j}+j-i-1 \\
j-i+1
\end{array}\right)\right]_{1 \leq i, j \leq n} .
$$

We explain after the proof of Theorem 12 how these formulae (5) and (6) can be deduced from a result of MacMahon on the enumeration of plane partitions.

In Section 2 we deduce the following special evaluations of the volume polynomial from some well-known results in the theory of empirical distributions: for $a, b \geq 0$,

$$
n ! V_{n}(a, b, \ldots, b)=a(a+n b)^{n-1},
$$

while for $n \geq 3$ and $a, b, c \geq 0$,

$$
n ! V_{n}(a, \overbrace{b, \ldots, b}^{n-2 \text { places }}, c)=a(a+n b)^{n-1}+n a(c-b)(a+(n-1) b)^{n-2},
$$

and for $n \geq 3,1 \leq m \leq n-2$ and $a, b, c \geq 0$,

$$
\begin{aligned}
& n ! V_{n}(a, \overbrace{b, \ldots, b}^{n-m-1 \text { places }} c, \overbrace{0, \ldots, 0}^{m-1 \text { places }}) \\
& \quad=a \sum_{j=0}^{m}\left(\begin{array}{l}
n \\
j
\end{array}\right)(c-(m+1-j) b)^{j}(a+(n-j) b)^{n-j-1} .
\end{aligned}
$$

As we indicate in Section 5, these formulae read from the theory of empirical distributions have interesting combinatorial interpretations in terms of parking functions and plane partitions.

\section{Uniform Order Statistics and Empirical Distribution Functions}

Let $\left(U_{n, i}, 1 \leq i \leq n\right)$ be the order statistics of $n$ independent uniform $(0,1)$ variables $U_{1}, U_{2}, \ldots, U_{n}$. That is to say, $U_{n, 1} \leq U_{n, 2} \leq \cdots \leq U_{n, n}$ are the ranked values of the $U_{i}, 1 \leq i \leq n$. Because the random vectors $\left(U_{n, j}, 1 \leq j \leq n\right)$ and $\left(1-U_{n, n+1-j}, 1 \leq\right.$ $j \leq n$ ) have the same uniform distribution with constant density $n$ ! on the simplex

$$
\left\{\boldsymbol{u} \in \mathbb{R}^{n}: 0 \leq u_{1} \leq \cdots \leq u_{n} \leq 1\right\}
$$

for arbitrary vectors $\boldsymbol{r}$ and $s$ in this simplex there are the formulae

$$
\begin{gathered}
P\left(U_{n, j} \leq s_{j} \text { for all } 1 \leq j \leq n\right)=n ! V_{n}\left(x_{1}, \ldots, x_{n}\right) \\
\text { where } \quad x_{j}:=s_{j}-s_{j-1},
\end{gathered}
$$

where $s_{0}:=0$ and

$$
\begin{gathered}
P\left(U_{n, j} \geq r_{j} \text { for all } 1 \leq j \leq n\right)=n ! V_{n}\left(x_{1}, \ldots, x_{n}\right) \\
\text { where } \quad x_{j}:=r_{n+2-j}-r_{n+1-j},
\end{gathered}
$$


where $r_{n+1}:=1$. Thus the probability

$$
P_{n}(\boldsymbol{r}, \boldsymbol{s}):=P\left(r_{j} \leq U_{n, j} \leq s_{j} \text { for all } 1 \leq j \leq n\right)
$$

can be evaluated in terms of $V_{n}$ if either $\boldsymbol{r}=\mathbf{0}$ or $\boldsymbol{s}=\mathbf{1}$. See Section 9.3 of [30] for a review of results involving these probabilities, including various recursion formulae which are useful for their computation.

Proof of Theorem 1. By homogeneity of $V_{n}$, it suffices to prove the formula when $s_{n} \leq 1$. Fix $\boldsymbol{x}$ and consider the probability (11). For $1 \leq i \leq n+1$ let $N_{i}$ denote the number of $U_{n, j}$ that fall in the interval $\left(s_{i-1}, s_{i}\right]$, with the conventions $s_{0}=0$ and $s_{n+1}=1$ :

$$
N_{i}:=\sum_{i=1}^{n} 1\left(s_{i-1}<U_{n, j} \leq s_{i}\right)=\sum_{i=1}^{n} 1\left(s_{i-1}<U_{j} \leq s_{i}\right) .
$$

The second expression for $N_{i}$ shows that the random vector $\left(N_{i}, 1 \leq i \leq n+1\right)$ has the multinomial distribution with parameters $n$ and $\left(x_{1}, \ldots, x_{n}, x_{n+1}\right)$ for $x_{i}:=s_{i}-s_{i-1}$, meaning that for each vector of $n+1$ nonnegative integers $\left(k_{i}, 1 \leq i \leq n+1\right)$ with $\sum_{i=1}^{n+1} k_{i}=n$, we have

$$
P\left(N_{i}=k_{i}, 1 \leq i \leq n+1\right)=n ! \prod_{i=1}^{n+1} \frac{x_{i} k_{i}}{k_{i} !}
$$

By definition of the $U_{n, j}$ and (14), the events $\left(U_{n, j} \leq s_{j}\right)$ and $\left(\sum_{i=1}^{j} N_{i} \geq j\right)$ are identical. Thus

$$
\begin{aligned}
P\left(U_{n, j} \leq s_{j} \text { for all } 1 \leq j \leq n\right) & \\
= & P\left(\sum_{i=1}^{j} N_{i} \geq j \text { for all } 1 \leq j \leq n\right) \\
& =\sum_{k \in K_{n}} P\left(N_{i}=k_{i}, 1 \leq i \leq n, N_{n+1}=0\right)=n ! \sum_{k \in K_{n}} \prod_{i=1}^{n} \frac{x_{i} k_{i}}{k_{i} !}
\end{aligned}
$$

by application of (15) with $k_{n+1}=0$. Compare the result of this calculation with (11) to obtain (2).

It is easily seen that the decomposition of the event (11) considered in the above argument corresponds to a polytopal subdivision of $\Pi_{n}(\boldsymbol{x})$ which for $n=2$ and $n=3$ is that shown in the right-hand panels of Figs. 1 and 2. See Section 4 for further discussion of this subdivision of $\Pi_{n}(\boldsymbol{x})$.

The following corollary of Theorem 1 spells out two more probabilistic interpretations of $V_{n}$.

Corollary 2. Let $\left(N_{i}, 1 \leq i \leq n+1\right)$ be a random vector with multinomial distribution with parameters $n$ and $\left(p_{1}, \ldots, p_{n+1}\right)$, as if $N_{i}$ is the number of times $i$ appears in 
a sequence of $n$ independent trials with probability $p_{i}$ of getting $i$ on each trial for $1 \leq i \leq n+1$, where $\sum_{i=1}^{n+1} p_{i}=1$. Then

$$
P\left(\sum_{j=1}^{i} N_{j} \geq i \text { for all } 1 \leq i \leq n\right)=n ! V_{n}\left(p_{1}, p_{2}, \ldots, p_{n}\right)
$$

and

$$
P\left(\sum_{j=1}^{i} N_{j}<i \text { for all } 1 \leq i \leq n\right)=n ! V_{n}\left(p_{n+1}, p_{n}, \ldots, p_{2}\right)
$$

Proof. The first formula is read from the previous proof of (2). The second is just the first applied to $\left(\hat{N}_{1}, \ldots, \hat{N}_{n+1}\right):=\left(N_{n+1}, \ldots, N_{1}\right)$ instead of $\left(N_{1}, \ldots, N_{n+1}\right)$, because

$$
\sum_{i=1}^{j} \hat{N}_{i}=\sum_{i=1}^{j} N_{n+2-i}=n-\sum_{i=1}^{n+1-j} N_{i}
$$

so that

$$
\sum_{i=1}^{j} \hat{N}_{i} \geq j \quad \text { iff } \quad \sum_{i=1}^{n+1-j} N_{i}<n+1-j,
$$

and hence the event that $\sum_{i=1}^{j} \hat{N}_{i} \leq j$ for all $1 \leq j \leq n$ is identical to the event that $\sum_{i=1}^{m} N_{i}<m$ for all $1 \leq m \leq n$.

Let

$$
F_{n}(t):=\frac{1}{n} \sum_{i=1}^{n} 1\left(U_{i} \leq t\right)=\frac{1}{n} \sum_{i=1}^{n} 1\left(U_{n, i} \leq t\right)
$$

be the usual empirical distribution function associated with the uniform random sample $U_{1}, \ldots, U_{n}$. So $F_{n}$ rises by a step of $1 / n$ at each of the sample points. It is well known [30] that for any for continuous increasing functions $f$ and $g$, the probability

$$
P\left(f(t) \leq F_{n}(t) \leq g(t) \text { for all } t\right)
$$

equals $P_{n}(\boldsymbol{r}, \boldsymbol{s})$ as in (13) where $\boldsymbol{r}$ and $\boldsymbol{s}$ are easily expressed in terms of values of the inverse functions of $f$ and $g$ at $i / n$ for $0 \leq i \leq n$. As an example, Daniels [3] discovered the remarkable fact that for $0 \leq p \leq 1$ the probability that the empirical distribution function does not cross the line joining $(0,0)$ to $(p, 1)$ equals $1-p$, no matter what $n=1,2, \ldots$ :

$$
P\left(F_{n}(t) \leq t / p \text { for all } 0 \leq t \leq 1\right)=1-p
$$

which can be rewritten as

$$
P\left(U_{n, i} \geq i p / n \text { for all } 1 \leq i \leq n\right)=1-p .
$$

As observed in Chapter X of [24], Daniels' formula (18) can be understood without calculation by an argument which gives the stronger result of Tákacs [38, Theorem 13.1] 
that this formula holds with $F_{n}$ replaced by $F$ for any random right-continuous nondecreasing step function $F$ with cyclically exchangeable increments and $F(0)=0$ and $F(1)=1$. Essentially, this is a continuous parameter form of the ballot theorem. Many other proofs of Daniels' formula are known: see Section 9.1 of [30] and papers cited there. The form (19) of Daniels' formula is equivalent via (12) to

$$
n ! V_{n}(1-p, p / n, \ldots, p / n)=1-p
$$

for $0 \leq p \leq 1$. By homogeneity of $V_{n}$, this amounts to the identity (7) of polynomials in two variables $a$ and $b$.

Pyke [25, Lemma 1] found the following formula: for all real $b$ and $x$ with

$$
\begin{gathered}
0 \leq b \leq 1 \quad \text { and } 0 \leq n b-x \leq 1, \\
P\left(\max _{1 \leq i \leq n}\left(b i-U_{n, i}\right) \leq x\right)=(1+x-n b) \sum_{j=0}^{\lfloor x / a\rfloor}\left(\begin{array}{c}
n \\
j
\end{array}\right)(j b-x)^{j}(1+x-j b)^{n-j-1} .
\end{gathered}
$$

As indicated in Exercise 2 on p. 354 of [30], this formula gives an expression for the probability that the empirical cumulative distribution function based on a sample of $n$ independent uniform $(0,1)$ variables crosses an arbitrary straight line through the unit square. See Section 9.1 of [30] for proof of an equivalent of (22), various related results, and further references. The identity in distribution

$$
\left(U_{n, i}, 1 \leq i \leq n\right) \stackrel{d}{=}\left(1-U_{n, n+1-i}, 1 \leq i \leq n\right)
$$

shows that the probability in (22) equals

$$
P\left(U_{n, i} \leq 1+x-n b+b(i-1) \text { for all } 1 \leq i \leq n\right)
$$

which according to (11) is equal in turn to

$$
\begin{aligned}
& n ! V_{n}\left(x_{1}, \ldots, x_{n}\right) \quad \text { for } \\
& \qquad x_{i}= \begin{cases}1+x-n b & \text { if } i=1, \\
b & \text { if } 2 \leq i<n-\lfloor x / a\rfloor+1, \\
(n-i+2) b-x & \text { if } i=n-\lfloor x / a\rfloor+1, \\
0 & \text { if } i>n-\lfloor x / a\rfloor+1 .\end{cases}
\end{aligned}
$$

For $a:=1+x-n b$ and $b$ subject to (21), that is $0<a \leq 1$ and $0 \leq b \leq 1$, the above discussion gives us equality of (22) and (24) with $x=a+n b-1$. In particular, provided $0 \leq x<a$ there is only a term for $j=0$ in (22), so the equality of (22) and (24) reduces to (7). Similarly, for $a \leq x<2 a$ there are only terms for $j=0$ and $j=1$ in (22). For $n \geq 3$ this allows us to deduce (8) from (22) first for $a, b, c>0$ with $a+(n-2) b+c=1$ and $c<b$, thence as an identity of polynomials in $a, b, c$. Similarly, for $n \geq 3$ and $1 \leq m \leq n-2$ when $\lfloor x / a\rfloor=m$ we obtain the identity (9) of polynomials in $a, b, c$.

According to Steck [36], [37], for $\boldsymbol{r}, \boldsymbol{s}$ in the simplex (10) there is the following determinantal formula for $P_{n}(\boldsymbol{r}, \boldsymbol{s})$ as in (13):

$$
P_{n}(\boldsymbol{r}, \boldsymbol{s})=n ! \operatorname{det}\left[\frac{1(j-i+1 \geq 0)}{(j-i+1) !}\left(s_{i}-r_{j}\right)_{+}^{j-i+1}\right]_{1 \leq i, j \leq n} .
$$


The special case of (5) when $s_{n} \leq 1$ can be read from (11), (13) and the special case of (25) with $\boldsymbol{r}=\mathbf{0}$ and $\boldsymbol{s}$ the vector of partial sums of $\boldsymbol{x}$. The general case of (5) follows by homogeneity of $V_{n}$ from the special case, with $x_{i}$ replaced by $x_{i} / \sigma$ for arbitrary $\sigma \geq \sum_{i=1}^{n} x_{i}$. See also [22], where probabilities of the form (25) are expressed in terms of Sheffer polynomials.

\section{Sections of Order Cones}

We obtain some results for a class of polytopes we call "sections of order cones" and then show in the next section how these results apply directly to $\Pi_{n}(\boldsymbol{x})$. Let $P$ be a partial ordering of the set $\left\{\alpha_{1}, \ldots, \alpha_{p}\right\}$, such that if $\alpha_{i}<\alpha_{j}$, then $i<j$. A linear extension of $P$ is an order-preserving bijection $\pi: P \rightarrow[p]=\{1,2, \ldots, p\}$, so if $z<z^{\prime}$ in $P$, then $\pi(z)<\pi\left(z^{\prime}\right)$. We identify $\pi$ with the permutation (written as a word) $a_{1} \cdots a_{p}$ of $[p]$ defined by $\pi\left(\alpha_{a_{i}}\right)=i$. In particular, the identity permutation $12 \cdots p$ is a linear extension of $P$. Let $\mathcal{L}(P)$ denote the set of linear extensions of $P$. Given $\pi=a_{1} \cdots a_{p} \in \mathcal{L}(P)$ define $\mathcal{A}_{\pi}$ to be the set of all order-preserving maps $f: P \rightarrow \mathbb{R}$ such that

$$
\begin{aligned}
& f\left(\alpha_{a_{1}}\right) \leq f\left(\alpha_{a_{2}}\right) \leq \cdots \leq f\left(\alpha_{a_{p}}\right), \\
& f\left(\alpha_{a_{j}}\right)<f\left(\alpha_{a_{j+1}}\right), \quad \text { if } \quad a_{j}>a_{j+1} .
\end{aligned}
$$

A basic property of order-preserving maps $f: P \rightarrow \mathbb{R}$ is given by the following theorem, which is equivalent to Lemma 4.5.3(a) of [32].

Theorem 3. The set of all order-preserving maps $f: P \rightarrow \mathbb{R}$ is a disjoint union of the sets $\mathcal{A}_{\pi}$ as $\pi$ ranges over $\mathcal{L}(P)$.

For instance, if $P$ is given by Fig. 3, then the order-preserving maps $f: P \rightarrow \mathbb{R}$ are partitioned by the following seven conditions:

$$
\begin{aligned}
& f\left(\alpha_{1}\right) \leq f\left(\alpha_{2}\right) \leq f\left(\alpha_{3}\right) \leq f\left(\alpha_{4}\right) \leq f\left(\alpha_{5}\right) \leq f\left(\alpha_{6}\right), \\
& f\left(\alpha_{1}\right) \leq f\left(\alpha_{2}\right) \leq f\left(\alpha_{3}\right) \leq f\left(\alpha_{5}\right)<f\left(\alpha_{4}\right) \leq f\left(\alpha_{6}\right), \\
& f\left(\alpha_{1}\right) \leq f\left(\alpha_{3}\right)<f\left(\alpha_{2}\right) \leq f\left(\alpha_{4}\right) \leq f\left(\alpha_{5}\right) \leq f\left(\alpha_{6}\right), \\
& f\left(\alpha_{1}\right) \leq f\left(\alpha_{3}\right)<f\left(\alpha_{2}\right) \leq f\left(\alpha_{5}\right)<f\left(\alpha_{4}\right) \leq f\left(\alpha_{6}\right), \\
& f\left(\alpha_{1}\right) \leq f\left(\alpha_{3}\right) \leq f\left(\alpha_{5}\right)<f\left(\alpha_{2}\right) \leq f\left(\alpha_{4}\right) \leq f\left(\alpha_{6}\right),
\end{aligned}
$$

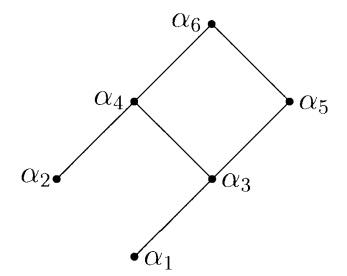

Fig. 3. A partially ordered set. 


$$
\begin{aligned}
& f\left(\alpha_{2}\right)<f\left(\alpha_{1}\right) \leq f\left(\alpha_{3}\right) \leq f\left(\alpha_{4}\right) \leq f\left(\alpha_{5}\right) \leq f\left(\alpha_{6}\right), \\
& f\left(\alpha_{2}\right)<f\left(\alpha_{1}\right) \leq f\left(\alpha_{3}\right) \leq f\left(\alpha_{5}\right)<f\left(\alpha_{4}\right) \leq f\left(\alpha_{6}\right) .
\end{aligned}
$$

Define the order cone $\mathcal{C}(P)$ of the poset $P$ to be the set of all order-preserving maps $f: P \rightarrow \mathbb{R}_{\geq 0}$. Thus $\mathcal{C}(P)$ is a pointed polyhedral cone in the space $\mathbb{R}^{P}$. Assume now that $P$ has a unique maximal element $\hat{1}$, and let $t_{1}<\cdots<t_{n}=\hat{1}$ be a chain $C$ in $P$. (With a little more work we could relax the assumption that $C$ is a chain. The condition that $t_{n}=\hat{1}$ entails no real loss of generality since we can just adjoin a $\hat{1}$ to $P$ and include it in $C$.) Let $x_{1}, \ldots, x_{n}$ be nonnegative real numbers. Set $u_{i}=x_{1}+\cdots+x_{i}$ and $\boldsymbol{u}=\left(u_{1}, \ldots, u_{n}\right)$. Let $W_{u}$ denote the subspace of $\mathbb{R}^{P}$ defined by $f\left(t_{i}\right)=u_{i}$ for $1 \leq i \leq n$. Define the order cone section $\mathcal{C}_{C}(P, \boldsymbol{u})$ to be the intersection $\mathcal{C}(P) \cap W_{\boldsymbol{u}}$, restricted to the coordinates $P-C$. (The restriction to the coordinates $P-C$ merely deletes constant coordinates and has no effect on the geometric and combinatorial structure of $\mathcal{C}(P) \cap W_{\boldsymbol{u}}$.) Equivalently, $\mathcal{C}_{C}(P, \boldsymbol{u})$ is the set of all order-preserving maps $f: P-C \rightarrow \mathbb{R}_{\geq 0}$ such that the extension of $f$ to $P$ defined by $f\left(t_{i}\right)=u_{i}$ remains order-preserving. Note that $\mathcal{C}_{C}(P, \boldsymbol{u})$ is bounded since for all $s \in P-C$ and all $f \in \mathcal{C}_{C}(P, \boldsymbol{u})$ we have $0 \leq f(s) \leq u_{n}$. Thus $\mathcal{C}_{C}(P, \boldsymbol{u})$ is a convex polytope contained in $\mathbb{R}^{P-C}$. Moreover, $\operatorname{dim} \mathcal{C}_{C}(P, \boldsymbol{u})=|P-C|$ provided each $x_{i}>0$ (or in certain other situations, such as when no element of $P-C$ is greater than $t_{1}$ ).

There is an alternative way to view the polytope $\mathcal{C}_{C}(P, \boldsymbol{u})$. Let $\mathcal{P}_{1}, \ldots, \mathcal{P}_{n}$ be convex polytopes (or just convex bodies) in the same ambient space $\mathbb{R}^{m}$, and let $x_{1}, \ldots, x_{n} \in$ $\mathbb{R}_{\geq 0}$. Define the Minkowski sum (or, more accurately, Minkowski linear combination)

$$
x_{1} \mathcal{P}_{1}+\cdots+x_{n} \mathcal{P}_{n}=\left\{x_{1} X_{1}+\cdots+x_{n} X_{n}: X_{i} \in \mathcal{P}_{i}\right\} .
$$

Then $\mathcal{Q}=x_{1} \mathcal{P}_{1}+\cdots+x_{n} \mathcal{P}_{n}$ is a convex polytope that was first investigated by Minkowski (at least for $m \leq 3$ ) and whose study belongs to the subject of integral geometry (e.g., [29]). In particular, the $m$-dimensional volume of $\mathcal{Q}$ has the form

$$
\operatorname{Vol}(\mathcal{Q})=\sum_{\substack{a_{1}+\ldots+a_{n}=m \\
a_{i} \in \mathbb{N}}}\left(\begin{array}{c}
m \\
a_{1}, \ldots, a_{n}
\end{array}\right) V\left(\mathcal{P}_{1}^{a_{1}}, \ldots, \mathcal{P}_{n}^{a_{n}}\right) x_{1}^{a_{1}} \cdots x_{n}^{a_{n}},
$$

where $V\left(\mathcal{P}_{1}^{a_{1}}, \ldots, \mathcal{P}_{n}^{a_{n}}\right) \in \mathbb{R}_{\geq 0}$. These numbers are known as the mixed volumes of the polytopes $\mathcal{P}_{1}, \ldots, \mathcal{P}_{n}$ and have been extensively investigated.

Now suppose that $\mathcal{P}_{1}, \ldots, \mathcal{P}_{n}$ are integer polytopes (i.e., their vertices have integer coordinates) in $\mathbb{R}^{m}$, and let $x_{1}, \ldots, x_{n} \in \mathbb{N}$. Given any integer polytope $\mathcal{P} \subset \mathbb{R}^{m}$, write

$$
N(\mathcal{P})=\#\left(\mathcal{P} \cap \mathbb{Z}^{m}\right),
$$

the number of integer points in $\mathcal{P}$. Then we call $N\left(x_{1} \mathcal{P}_{1}+\cdots+x_{n} \mathcal{P}_{n}\right)$, regarded as a function of $x_{1}, \ldots, x_{n} \in \mathbb{N}$, the mixed lattice point enumerator of $\mathcal{P}_{1}, \ldots, \mathcal{P}_{n}$. It was shown by McMullen [16] (see also [17] and [18] for two related survey articles) that $N\left(x_{1} \mathcal{P}_{1}+\cdots+x_{n} \mathcal{P}_{n}\right)$ is a polynomial in $x_{1}, \ldots, x_{n}$ (with rational coefficients) of total degree at most $m$. Moreover, the terms of degree $m$ are given by $\operatorname{Vol}\left(x_{1} \mathcal{P}_{1}+\cdots+x_{n} \mathcal{P}_{n}\right)$. Hence the coefficients of the terms of degree $m$ are nonnegative, but in general the coefficients of $N\left(x_{1} \mathcal{P}_{1}+\cdots+x_{n} \mathcal{P}_{n}\right)$ may be negative. In the special case $n=1$, the 
mixed lattice point enumerator $N(x \mathcal{P})$ is called the Ehrhart polynomial of the integer polytope $\mathcal{P}$ and is denoted $i(\mathcal{P}, x)$. An introduction to Ehrhart polynomials appears on pp. 235-241 of [32].

Define the order polytope $\mathcal{O}(P)$ of the finite poset $P$ to be the set of all orderpreserving maps $f: P \rightarrow[0,1]=\{x \in \mathbb{R}: 0 \leq x \leq 1\}$. Thus $\mathcal{O}(P)$ is a convex polytope in $\mathbb{R}^{P}$ of dimension $|P|$. The basic properties of order polytopes are developed in [31].

Theorem 4. Given $P, C$, and $\boldsymbol{u}$ as above, so $u_{i}=x_{1}+\cdots+x_{i}$, let

$$
P_{i}=\left\{s \in P-C: s \nless t_{i-1}\right\}
$$

(with $P_{1}=P-C$ ). Regard the order polytope $\mathcal{O}\left(P_{i}\right)$ as lying in $\mathbb{R}^{P-C}$ by setting coordinates indexed by elements of $(P-C)-P_{i}$ equal to 0 . Then

$$
\mathcal{C}_{C}(P, \boldsymbol{u})=x_{1} \mathcal{O}\left(P_{1}\right)+x_{2} \mathcal{O}\left(P_{2}\right)+\cdots+x_{n} \mathcal{O}\left(P_{n}\right)
$$

Proof. We can regard $\mathcal{O}\left(P_{i}\right)$ as the set of order-preserving maps $f: P-C \rightarrow[0,1]$ such that $f(s)=0$ if $s<t_{i-1}$. From this it is clear that every element of $x_{1} \mathcal{O}\left(P_{1}\right)+$ $x_{2} \mathcal{O}\left(P_{2}\right)+\cdots+x_{n} \mathcal{O}\left(P_{n}\right)$ is an order-preserving map $g: P-C \rightarrow \mathbb{R}_{\geq 0}$ such that the extension of $g$ to $P$ defined by $g\left(t_{i}\right)=x_{1}+\cdots+x_{i}$ remains order-preserving. Hence

$$
\mathcal{C}_{C}(P, \boldsymbol{u}) \supseteq x_{1} \mathcal{O}\left(P_{1}\right)+x_{2} \mathcal{O}\left(P_{2}\right)+\cdots+x_{n} \mathcal{O}\left(P_{n}\right) .
$$

For the converse, we may assume (by deleting elements of $P$ if necessary) that each $x_{i}>$ 0 . Let $f \in \mathcal{C}_{C}(P, \boldsymbol{u})$. Let $s \in P_{C}$ and define $g_{1}(s)=f(s)$ and $f_{1}(s)=\min \left(1, x_{1}^{-1} g_{1}(s)\right)$. Set

$$
g_{2}(s)=g_{1}(s)-x_{1} f_{1}(s)=\max \left(g_{1}(s)-x_{1}, 0\right) .
$$

Now let $f_{2}(s)=\min \left(1, x_{2}^{-1} g_{2}(s)\right)$ and set

$$
g_{3}(s)=g_{2}(s)-x_{2} f_{2}(s)=\max \left(g_{2}(s)-x_{2}, 0\right) .
$$

Continuing in this way gives functions $f_{1}, f_{2}, \ldots, f_{n}$, for which it can be checked that $f_{i} \in \mathcal{O}\left(P_{i}\right)$ and

$$
f=x_{1} f_{1}+\cdots+x_{n} f_{n},
$$

so

$$
\mathcal{C}_{C}(P, \boldsymbol{u}) \subseteq x_{1} \mathcal{O}\left(P_{1}\right)+x_{2} \mathcal{O}\left(P_{2}\right)+\cdots+x_{n} \mathcal{O}\left(P_{n}\right)
$$

We now want to give a formula for the number of integer points in $\mathcal{C}_{C}(P, \boldsymbol{u})$, which by Theorem 4 is just the mixed lattice point enumerator of the polytopes $\mathcal{O}\left(P_{i}\right)$. Let $C$ be the chain $t_{1}<\cdots<t_{n}=\hat{1}$ as above. Given $\pi=a_{1} \cdots a_{p} \in \mathcal{L}(P)$, write $h_{i}(\pi)$ for the height of $t_{i}$ in $\pi$, i.e., $t_{i}=\pi^{-1}\left(a_{h_{i}(\pi)}\right)$. Thus $1 \leq h_{1}(\pi)<\cdots<h_{n}(\pi)=p$. Also write

$$
d_{i}(\pi)=\#\left\{j: h_{i-1}(\pi) \leq j<h_{i}(\pi), a_{j}>a_{j+1}\right\},
$$


where we set $h_{0}(\pi)=0$ and $a_{0}=0$. Thus $d_{i}(\pi)$ is the number of descents of $\pi$ appearing between $h_{i-1}(\pi)$ and $h_{i}(\pi)$. Recall (e.g., Section 1.2 of [32]) that the number of ways to choose $j$ objects with repetition from a set of $k$ objects is given by

$$
\left.\left(\begin{array}{l}
k \\
j
\end{array}\right)\right)=\left(\begin{array}{c}
k+j-1 \\
j
\end{array}\right)=\frac{k(k+1) \cdots(k+j-1)}{j !} .
$$

$\operatorname{Regarding}\left(\left(\begin{array}{l}k \\ j\end{array}\right)\right)$ as a polynomial in $k \in \mathbb{Z}$, note that $\left(\left(\begin{array}{l}k \\ j\end{array}\right)\right)=0$ for $-j+1 \leq k \leq 0$.

Theorem 5. We have

$$
N\left(\mathcal{C}_{C}(P, \boldsymbol{u})\right)=\sum_{\pi \in \mathcal{L}(P)} \prod_{i=1}^{n-1}\left(\left(\begin{array}{c}
x_{i}-d_{i}(\pi)+1 \\
h_{i}(\pi)-h_{i-1}(\pi)-1
\end{array}\right)\right) .
$$

Proof. Fix $\pi=a_{1} \cdots a_{p} \in \mathcal{L}(P)$. Write $h_{i}=h_{i}(\pi)$ and $d_{i}=d_{i}(\pi)$. Let $f: P \rightarrow \mathbb{R}$ be an order-preserving map such that (a) $f \in \mathcal{A}_{\pi}$, (b) $f\left(t_{i}\right)=u_{i}=x_{1}+\cdots+x_{i}$, and (c) the restriction $\left.f\right|_{P-C}$ of $f$ to $P-C$ satisfies $\left.f\right|_{P-C} \in \mathcal{C}_{C}(P, \boldsymbol{u})$. If we write $c_{i}=f\left(\alpha_{a_{i}}\right)$, then for fixed $\pi$ it follows from Theorem 3 that the integer points $\left.f\right|_{P-C} \in \mathcal{C}_{C}(P, \boldsymbol{u})$, where $f$ satisfies (a) and (b), are given by

$$
\begin{gathered}
0 \leq c_{1} \leq c_{2} \leq \cdots \leq c_{h_{1}}=x_{1} \leq c_{h_{1}+1} \leq \cdots \leq c_{h_{2}} \\
=x_{1}+x_{2} \leq \cdots \leq c_{p}=x_{1}+\cdots+x_{n}, \\
c_{j}<c_{j+1} \quad \text { if } a_{j}>a_{j+1} .
\end{gathered}
$$

Let $\alpha, \beta, m \in \mathbb{N}$ and $0 \leq j_{1}<j_{2}<\cdots<j_{q} \leq m$. Elementary combinatorial reasoning shows that the number of integer vectors $\left(r_{1}, \ldots, r_{m}\right)$ satisfying

$$
\begin{gathered}
\alpha=r_{0} \leq r_{1} \leq \cdots \leq r_{m} \leq r_{m+1}=\alpha+\beta, \\
r_{j_{i}}<r_{j_{i+1}} \quad \text { for } \quad 1 \leq i \leq q
\end{gathered}
$$

is equal to $\left(\left(\begin{array}{c}\beta-q+1 \\ m\end{array}\right)\right)$. Hence the number of integer sequences satisfying (29) and (30) is given by

$$
\left(\left(\begin{array}{c}
x_{1}-d_{1}+1 \\
h_{1}-1
\end{array}\right)\right)\left(\left(\begin{array}{c}
x_{2}-d_{2}+1 \\
h_{2}-h_{1}-1
\end{array}\right)\right) \cdots\left(\left(\begin{array}{c}
x_{n}-d_{n}+1 \\
h_{n}-h_{n-1}-1
\end{array}\right)\right) .
$$

Summing over all $\pi \in \mathcal{L}(P)$ yields (28).

Example 6. Let $P$ be given by Fig. 3, and let $t_{1}=\alpha_{1}, t_{2}=\alpha_{3}$, and $t_{3}=\alpha_{6}$. The conditions in (26) become in the notation of the above proof as follows:

$$
\begin{aligned}
& 0 \leq c_{1}=x_{1} \leq c_{2} \leq c_{3}=x_{1}+x_{2} \leq c_{4} \leq c_{5} \leq c_{6}=x_{1}+x_{2}+x_{3}, \\
& 0 \leq c_{1}=x_{1} \leq c_{2} \leq c_{3}=x_{1}+x_{2} \leq c_{4}<c_{5} \leq c_{6}=x_{1}+x_{2}+x_{3}, \\
& 0 \leq c_{1}=x_{1} \leq c_{2}=x_{1}+x_{2}<c_{3} \leq c_{4} \leq c_{5} \leq c_{6}=x_{1}+x_{2}+x_{3},
\end{aligned}
$$




$$
\begin{aligned}
& 0 \leq c_{1}=x_{1} \leq c_{2}=x_{1}+x_{2}<c_{3} \leq c_{4}<c_{5} \leq c_{6}=x_{1}+x_{2}+x_{3}, \\
& 0 \leq c_{1}=x_{1} \leq c_{2}=x_{1}+x_{2} \leq c_{3}<c_{4} \leq c_{5} \leq c_{6}=x_{1}+x_{2}+x_{3}, \\
& 0 \leq c_{1}<c_{2}=x_{1} \leq c_{3}=x_{1}+x_{2} \leq c_{4} \leq c_{5} \leq c_{6}=x_{1}+x_{2}+x_{3}, \\
& 0 \leq c_{1}<c_{2}=x_{1} \leq c_{3}=x_{1}+x_{2} \leq c_{4}<c_{5} \leq c_{6}=x_{1}+x_{2}+x_{3},
\end{aligned}
$$

yielding

$$
\begin{aligned}
N\left(\mathcal{C}_{C}(P, \boldsymbol{u})\right)= & \left(\left(\begin{array}{c}
x_{2}+1 \\
1
\end{array}\right)\right)\left(\left(\begin{array}{c}
x_{3}+1 \\
2
\end{array}\right)\right)+\left(\left(\begin{array}{c}
x_{2}+1 \\
1
\end{array}\right)\right)\left(\left(\begin{array}{c}
x_{3} \\
2
\end{array}\right)\right)+\left(\left(\begin{array}{c}
x_{3} \\
3
\end{array}\right)\right) \\
& \left.\left.+\left(\left(\begin{array}{c}
x_{3}-1 \\
3
\end{array}\right)\right)+\left(\left(\begin{array}{c}
x_{3} \\
3
\end{array}\right)\right)+\left(\left(\begin{array}{c}
x_{1} \\
1
\end{array}\right)\right)\left(\begin{array}{c}
x_{3}+1 \\
2
\end{array}\right)\right)+\left(\left(\begin{array}{c}
x_{1} \\
1
\end{array}\right)\right)\left(\begin{array}{c}
x_{3} \\
2
\end{array}\right)\right) .
\end{aligned}
$$

We mentioned earlier that the terms of highest degree (here of degree $|P-C|$ ) of $N\left(x_{1} \mathcal{P}_{1}+\cdots+x_{n} \mathcal{P}_{n}\right)$ are given by $\operatorname{Vol}\left(x_{1} \mathcal{P}_{1}+\cdots+x_{n} \mathcal{P}_{n}\right)$. Hence we obtain from Theorem 5 the following result.

Corollary 7. The volume of $\mathcal{C}_{C}(P, \boldsymbol{u})$ is given by

$$
\operatorname{Vol}\left(\mathcal{C}_{C}(P, \boldsymbol{u})\right)=\sum_{\pi \in \mathcal{L}(P)} \prod_{i=1}^{n} \frac{x_{i}^{h_{i}(\pi)-h_{i-1}(\pi)}}{\left(h_{i}(\pi)-h_{i-1}(\pi)\right) !} .
$$

Thus if $m=|P-C|$, then the mixed volume $m ! \cdot V\left(\mathcal{O}\left(P_{1}\right)^{a_{1}}, \ldots, \mathcal{O}\left(P_{n}\right)^{a_{n}}\right)$ is equal to the number of linear extensions $\pi \in \mathcal{L}(P)$ such that t $i_{i}$ has height $a_{1}+\cdots+a_{i}$ in $\pi$, for $1 \leq i \leq n$.

The case $n=2$ of Corollary 7 (or equivalently the case $n=1$ where $t_{1}$ can be any element of $P$, not just the top element) appears in (16) of [31].

The product of two polytopes $\mathcal{P} \in \mathbb{R}^{p}$ and $\mathcal{Q} \in \mathbb{R}^{q}$ is defined to be their cartesian product $\mathcal{P} \times \mathcal{Q} \in \mathbb{R}^{p+q}$. If $\bar{L}(\mathcal{P})$ denotes the poset of nonempty faces of $\mathcal{P}$, then $\bar{L}(\mathcal{P} \times \mathcal{Q})=\bar{L}(\mathcal{P}) \times \bar{L}(\mathcal{Q})$ (see pp. 9-10 of [39]). If $\mathcal{P}$ is a $d$-simplex, then $\bar{L}(\mathcal{P})$ is just a boolean algebra of rank $d$ with the minimum element removed. Moreover, the product of $n$ one-dimensional simplices is combinatorially equivalent (even affinely equivalent) to a $d$-cube. If $\pi=a_{1} \cdots a_{p} \in \mathcal{L}_{P}$, then define $\Lambda_{\pi}$ to be the subset of $\mathcal{C}_{C}(P, \boldsymbol{u})$ given by (29). Thus when each $x_{i}>0$ we have that $\Lambda_{\pi}$ is a product of simplices of dimensions $h_{1}-1, h_{2}-h_{1}-1, \ldots, h_{p}-h_{1}-1$, and

$$
\operatorname{Vol}\left(\Lambda_{\pi}\right)=\prod_{i=1}^{n} \frac{x_{i}^{h_{i}(\pi)-h_{i-1}(\pi)}}{\left(h_{i}(\pi)-h_{i-1}(\pi)\right) !} .
$$

Moreover, the $\Lambda_{\pi}$ 's form the chambers of a polyhedral decomposition $\Omega_{C}(P, \boldsymbol{u})$ of $\mathcal{C}_{C}(P, \boldsymbol{u})$. We regard $\Omega_{C}(P, \boldsymbol{u})$ as the set of all faces of the $\Lambda_{\pi}$ 's (including the $\Lambda_{\pi}$ 's themselves), partially ordered by inclusion. Note that formula (31) corresponds to an explicit decomposition of $\mathcal{C}_{C}(P, \boldsymbol{u})$ into "nice" pieces (products of simplices) whose volumes are the terms in (31). 
Our next result concerns the combinatorial structure of the decomposition of $\mathcal{C}_{C}(P, \boldsymbol{u})$ into the chambers $\Lambda_{\pi}$. First we review some information from Section 5 of [31] about the cone $\mathcal{C}(P)$ of all order-preserving maps $f: P \rightarrow \mathbb{R}_{\geq 0}$. (Paper [31] actually deals with the order complex $\mathcal{O}(P)$ rather than the cone $\mathcal{C}(P)$, but this does not affect our arguments.) Recall (e.g., p. 100 of [32]) that an order ideal $I$ of $P$ is a subset of $P$ such that if $t \in I$ and $s<t$, then $s \in I$. The poset (actually a distributive lattice) of all order ideals of $P$, ordered by inclusion, is denoted $J(P)$. Given a chain $K: \emptyset=I_{0}<I_{1}<\cdots<I_{k}=P$ in $J(P)$, define $\mathcal{C}_{K}(P)$ to consist of all $f: P \rightarrow \mathbb{R}_{\geq 0}$ satisfying

$$
0 \leq f\left(I_{1}\right) \leq f\left(I_{2}-I_{1}\right) \leq \cdots \leq f\left(I_{k}-I_{k-1}\right)
$$

where $f(S)$ denotes the common value of $f$ at all the elements of the subset $S$ of $P$. Clearly, $\mathcal{C}_{K}(P)$ is a $k$-dimensional cone in $\mathbb{R}^{P}$. It is not hard to see that the set $\Omega(P)=\left\{\mathcal{C}_{K}(P)\right.$ : $K$ is a chain in $J(P)$ containing $\emptyset$ and $\left.P\right\}$ is a triangulation of $\mathcal{C}(P)$. The chambers (maximal faces) of $\Omega(P)$ consist of the cones

$$
0 \leq f\left(\alpha_{a_{1}}\right) \leq \cdots \leq f\left(\alpha_{a_{p}}\right)
$$

where $\pi=a_{1} \cdots a_{p} \in \mathcal{L}(P)$. Moreover, $\mathcal{C}_{K}(P)$ is an interior face of $\Omega(P)$ (i.e., does not lie on the boundary) if and only if each subset $I_{i}-I_{i-1}$ of (32) is an antichain, i.e., no two distinct elements of $I_{i}-I_{i-1}$ are comparable. Such chains of $J(P)$ are called Loewy chains. Let $\Omega^{\circ}(P)$ denote the set of interior faces of $\Omega(P)$ regarded as a partially ordered set under inclusion. Thus $\Omega^{\circ}(P)$ is isomorphic to the set of Loewy chains of $J(P)$, ordered by inclusion. Similarly, we let $\Omega_{C}^{\circ}(P, \boldsymbol{u})$ denote the set of interior faces of the polyhedral decomposition $\Omega_{C}(P, \boldsymbol{u})$.

Theorem 8. Let $W_{u}$ denote the subspace of $\mathbb{R}^{P}$ given by $f\left(t_{i}\right)=u_{i}, 1 \leq i \leq n$. Define a map $\phi: \Omega^{\circ}(P) \rightarrow \Omega_{C}^{\circ}(P, \boldsymbol{u})$ by letting $\phi\left(\mathcal{C}_{K}(P)\right)$ equal $\phi_{K}(P) \cap W_{\boldsymbol{u}}$ restricted to the coordinates $P-C$. Then $\phi$ is an isomorphism of posets.

Proof. Let (32) define an interior face $\mathcal{C}_{K}(P)$ of $\mathcal{C}(P)$, so $\emptyset=I_{0}<I_{1}<\cdots<I_{k}=P$ is a Loewy chain. Thus each set $I_{j}-I_{j-1}$ contains at most one element of the chain $C: t_{1}<\cdots<t_{n}$. Let $t_{i} \in I_{j_{i}}-I_{j_{i}-1}$. (In particular, $j_{n}=k$ since $t_{n}=\hat{1}$.) Then $\phi\left(\mathcal{C}_{K}(P)\right)$ is defined by the equations

$$
\begin{aligned}
0 & \leq f\left(I_{1}\right) \leq f\left(I_{2}-I_{1}\right) \leq \cdots \leq f\left(I_{j_{1}}-I_{j_{1}-1}\right)=u_{1} \\
& \leq f\left(I_{j_{1}+1}-I_{j_{1}}\right) \leq \cdots \leq f\left(I_{j_{2}}-I_{j_{2}-1}\right)=u_{2} \leq \cdots \leq f\left(I_{k}-I_{k-1}\right)=u_{n} .
\end{aligned}
$$

It follows immediately that $\phi$ is a bijection, and that two Loewy chains $K$ and $K^{\prime}$ satisfy $K \subseteq K^{\prime}$ if and only if $\phi\left(\mathcal{C}_{K}(P)\right) \subseteq \phi\left(\mathcal{C}_{K^{\prime}}(P)\right)$. Hence $\phi$ is a poset isomorphism.

The point of Theorem 8 is that it gives a simple combinatorial description (namely, the poset $\Omega^{\circ}(P)$, which is isomorphic to the set of Loewy chains of $J(P)$ under inclusion) of the geometrically defined poset $\Omega_{C}^{\circ}(P, \boldsymbol{u})$. Note that $\Omega^{\circ}(P)$ depends only on $P$, not on the chain $C$. 


\section{4. $\Pi_{n}(x)$ as a Section of an Order Cone}

In this section we apply the theory developed in the previous section to $\Pi_{n}(\boldsymbol{x})$. We say that two integer polytopes $\mathcal{P} \subset \mathbb{R}^{k}$ and $\mathcal{Q} \subset \mathbb{R}^{m}$ are integrally equivalent if there is an affine transformation $\varphi: \mathbb{R}^{k} \rightarrow \mathbb{R}^{m}$ whose restriction to $\mathcal{P}$ is a bijection $\varphi: \mathcal{P} \rightarrow \mathcal{Q}$, and such that if aff denotes affine span, then $\varphi$ restricted to $\mathbb{Z}^{k} \cap \operatorname{aff}(\mathcal{P})$ is a bijection $\varphi: \mathbb{Z}^{k} \cap \operatorname{aff}(\mathcal{P}) \rightarrow \mathbb{Z}^{m} \cap \operatorname{aff}(\mathcal{Q})$. It follows that $\mathcal{P}$ and $\mathcal{Q}$ have the same combinatorial type and the same "integral structure," and hence the same volume, Ehrhart polynomial, etc.

Now let $\boldsymbol{i}$ denote an $i$-element chain, and let $Q_{n}=\mathbf{2} \times \boldsymbol{n}$, the product of a twoelement chain with an $n$-element chain. We regard the elements of $Q_{n}$ as $\alpha_{1}, \ldots, \alpha_{2 n}$ with $\alpha_{1}<\cdots<\alpha_{n}, \alpha_{n+1}<\cdots<\alpha_{2 n}$, and $\alpha_{i}<\alpha_{n+i}$ for $1 \leq i \leq n$. Let $t_{i}=\alpha_{n+i}$, and let $C$ be the chain $t_{1}<\cdots<t_{n}$. As in the previous section let $x_{1}, \ldots, x_{n} \geq 0$, and set $u_{i}=x_{1}+\cdots+x_{i}$. The polytope $\mathcal{C}_{C}\left(Q_{n}, \boldsymbol{u}\right) \subset \mathbb{R}^{Q_{n}-C} \cong \mathbb{R}^{n}$, thus by definition is given by the equations

$$
\begin{gathered}
0 \leq f_{1} \leq \cdots \leq f_{n}, \\
f_{i} \leq u_{i}, \quad 1 \leq i \leq n .
\end{gathered}
$$

Let $y_{i}=f_{i}-f_{i-1}$ (with $f_{0}=0$ ). Then the above equations become

$$
\begin{gathered}
y_{i} \geq 0, \quad 1 \leq i \leq n, \\
y_{1}+\cdots+y_{i} \leq x_{1}+\cdots+x_{n} .
\end{gathered}
$$

These are just the equations for $\Pi_{n}(\boldsymbol{x})$. The transformation $y_{i}=f_{i}-f_{i-1}$ induces an integral equivalence between $\mathcal{C}_{C}\left(Q_{n}, \boldsymbol{u}\right)$ and $\Pi_{n}(\boldsymbol{u})$. Hence the results of the above section, when specialized to $P=Q_{n}$, are directly applicable to $\Pi_{n}(\boldsymbol{x})$.

Theorem 4 expresses $\mathcal{C}_{C}(P, \boldsymbol{u})$ as a Minkowski linear combination of order polytopes $\mathcal{O}\left(P_{i}\right)$. In the present situation, where $P=\mathbf{2} \times \boldsymbol{n}$, the poset $P_{i}$ is just the chain $\alpha_{i}<$ $\alpha_{i+1}<\cdots<\alpha_{n}$. The order polytope $\mathcal{O}\left(P_{i}\right)$ is defined by the conditions

$$
f_{1}=\cdots=f_{i-1}=0, \quad 0 \leq f_{i} \leq \cdots \leq f_{n} \leq 1 .
$$

This is just a simplex of dimension $n-i+1$ with vertices $\left(0^{j}, 1^{n-j}\right), i-1 \leq j \leq n$, where $\left(0^{j}, 1^{n-j}\right)$ denotes a vector of $j$ zeros followed by $n-j$ ones. Switching to the $y$ coordinates (i.e., $y_{i}=f_{i}-f_{i-1}$ ) yields the following result.

Theorem 9. Let $\tau_{i}$ be the $(n-i+1)$-dimensional simplex in $\mathbb{R}^{n}$ defined by

$$
\begin{gathered}
y_{1}=\cdots=y_{i-1}=0, \\
y_{i} \geq 0, \ldots, y_{n} \geq 0, \\
y_{i}+\cdots+y_{n} \leq 1,
\end{gathered}
$$

with vertices $\left(0^{j-1}, 1,0^{n-j}\right)$ for $i \leq j \leq n$, and $(0,0, \ldots, 0)$. Then

$$
\Pi_{n}(\boldsymbol{x})=x_{1} \tau_{1}+x_{2} \tau_{2}+\cdots+x_{n} \tau_{n}
$$


Consider the set $\mathcal{L}\left(Q_{n}\right)$ of linear extensions of $Q_{n}$. A linear extension $\pi=a_{1} \cdots a_{2 n} \in$ $\mathcal{L}\left(Q_{n}\right)$ is uniquely determined by the positions of $n+1, \ldots, 2 n$ (since $1, \ldots, n$ must appear in increasing order). If $a_{j_{i}}=n+i$ for $1 \leq i \leq n$, then $1 \leq j_{1}<\cdots<j_{n}=2 n$ and $j_{i} \geq 2 i$. The number of such sequences is just the Catalan number $C_{n}=(1 /(n+1))\left(\begin{array}{c}2 n \\ n\end{array}\right)$ (see, e.g., Exercise 6.19(t) of [34], which is a minor variation). If we set $k_{i}=j_{i}-j_{i-1}$ (with $j_{0}=0$ ), then the sequences $\boldsymbol{k}=\left(k_{1}, \ldots, k_{n}\right)$ are just those of (3). Moreover, in the linear extension $a_{1} \cdots a_{2 n}$ there are no descents to the left of $n+1$, and there is exactly one descent between $n+i$ and $n+i+1$ provided that $k_{i+1}-k_{i} \geq 2$. (If $k_{i+1}-k_{i}=1$, then there are no descents between $n+i$ and $n+i+1$.) By Theorem 5 we conclude

$$
N\left(\Pi_{n}(\boldsymbol{x})\right)=\sum_{k \in K_{n}}\left(\left(\begin{array}{c}
x_{1}+1 \\
k_{1}
\end{array}\right)\right) \prod_{i=2}^{n}\left(\left(\begin{array}{l}
x_{i} \\
k_{i}
\end{array}\right)\right)
$$

where $K_{n}$ is given by (3). Taking terms of highest degree yields Theorem 1 . Thus we have obtained an explicit decomposition of $\Pi_{n}(x)$ into products of simplices whose volumes are the terms in (2). (A completely different such decomposition will be given in Section 6.) Moreover, Theorem 8 gives the combinatorial structure of the interior faces of this decomposition.

Note. Equation (33) was obtained independently by Ira Gessel (private communication) by a different method.

We illustrate the above discussion with the case $n=3$. The poset $Q_{3}$ is shown in Fig. 4. The linear extensions of $Q_{3}$ are given as follows, with the elements 4, 5,6 corresponding to the chain $C$ shown in boldface:

123456

124356

124536

142356

142536

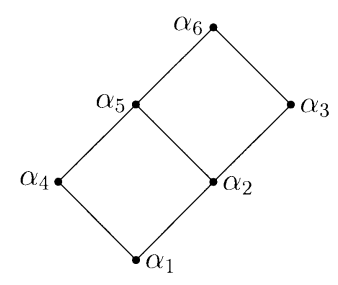

Fig. 4. The poset $Q_{3}=\mathbf{2} \times \mathbf{3}$. 


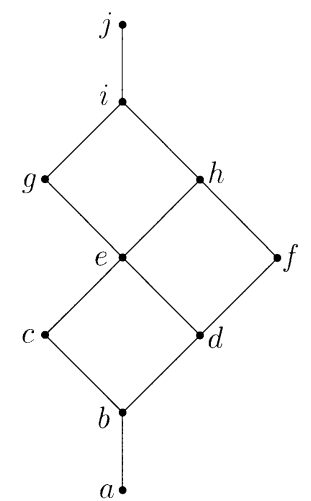

Fig. 5. The lattice $J\left(Q_{3}\right)$ of order ideals of $Q_{3}$.

Hence the points $\left(y_{1}, y_{2}, y_{3}\right) \in \Pi_{3}(\boldsymbol{x})$ are decomposed into the sets

$$
\begin{gathered}
0 \leq y_{1} \leq y_{2} \leq y_{3} \leq x_{1}, \\
0 \leq y_{1} \leq y_{2} \leq x_{1}<y_{3} \leq x_{1}+x_{2}, \\
0 \leq y_{1} \leq y_{2} \leq x_{1} \leq x_{1}+x_{2}<y_{3} \leq x_{1}+x_{2}+x_{3}, \\
0 \leq y_{1} \leq x_{1}<y_{2} \leq y_{3} \leq x_{1}+x_{2}, \\
0 \leq y_{1} \leq x_{1}<y_{2} \leq x_{1}+x_{2}<y_{3} \leq x_{1}+x_{2}+x_{3},
\end{gathered}
$$

yielding

$$
\begin{aligned}
N\left(\Pi_{3}(\boldsymbol{x})\right)= & \left(\left(\begin{array}{c}
x_{1}+1 \\
3
\end{array}\right)\right)+\left(\left(\begin{array}{c}
x_{1}+1 \\
2
\end{array}\right)\right)\left(\left(\begin{array}{c}
x_{2} \\
1
\end{array}\right)\right)+\left(\left(\begin{array}{c}
x_{1}+1 \\
2
\end{array}\right)\right)\left(\left(\begin{array}{c}
x_{3} \\
1
\end{array}\right)\right) \\
& \left.+\left(\left(\begin{array}{c}
x_{1}+1 \\
1
\end{array}\right)\right)\left(\left(\begin{array}{c}
x_{2} \\
2
\end{array}\right)\right)+\left(\left(\begin{array}{c}
x_{1}+1 \\
1
\end{array}\right)\right)\left(\left(\begin{array}{c}
x_{2} \\
1
\end{array}\right)\right)\left(\begin{array}{c}
x_{3} \\
1
\end{array}\right)\right) .
\end{aligned}
$$

Theorem 8 allows us to describe the incidence relations among the faces of the decomposition of $\Pi_{3}(\boldsymbol{x})$ whose chambers are the closures of the five sets in (34). The lattice $J\left(Q_{3}\right)$ of order ideals of $Q_{3}$ has five maximal chains. This lattice is shown in Fig. 5, with elements labeled $a, b, \ldots, j$. The elements $a, b, i, j$ appear in every Loewy chain of $J\left(Q_{3}\right)$ and can be ignored. The simplicial complex of chains of $J(P)$ (with $a, b, i, j$ removed) is shown in Fig. 6(a). The Loewy chains correspond to the interior faces, of which five have dimension 2, five have dimension 1 , and one has dimension 0 . Figure 6(b) shows the "dual complex" of the interior faces. This gives the incidence relations among

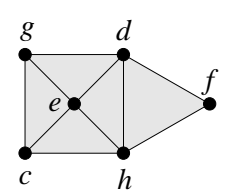

(a)

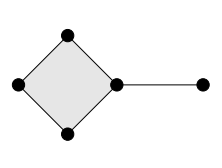

(b)

Fig. 6. The order complex of $J\left(Q_{3}\right)$ with $a, b, i, j$ omitted, and the interior face dual complex. 
the five chambers of the decomposition of $\Pi_{3}(\boldsymbol{x})$ into five products of simplices obtained from $\Omega_{C}^{\circ}(P, \boldsymbol{u})$ by the change of coordinates $y_{i}=f_{i}-f_{i-1}$ discussed above. For a picture, see the second subdivision of $\Pi_{3}(\boldsymbol{x})$ in Fig. 2.

We mentioned earlier that in general the coefficients of the mixed lattice point enumerator $N\left(x_{1} \mathcal{P}_{1}+\cdots+x_{n} \mathcal{P}_{n}\right)$ may be negative. The polytope $\Pi_{n}(\boldsymbol{x})$ is an exception, however, and in fact satisfies a slightly stronger property.

Corollary 10. The polynomial $N\left(\Pi_{n}\left(x_{1}-1, x_{2}, \ldots, x_{n}\right)\right)$ has nonnegative coefficients.

Proof. Immediate from (33), since the polynomial $\left(\begin{array}{c}t \\ i\end{array}\right)$ has nonnegative coefficients.

Note. One can also think of $\mathcal{C}_{C}\left(Q_{n}, \boldsymbol{u}\right)$ as the "polytope of fractional shapes contained in the shape $\left(u_{n}, u_{n-1}, \ldots, u_{1}\right)$." In general, let $\lambda=\left(\lambda_{1}, \ldots, \lambda_{n}\right)$ be a partition, i.e., $\lambda_{i} \in \mathbb{N}$ and $\lambda_{1} \geq \cdots \geq \lambda_{n}$, which we also call a shape. We say that a shape $\mu=\left(\mu_{1}, \ldots, \mu_{n}\right)$ is contained in $\lambda$ if $\mu_{i} \leq \lambda_{i}$ for all $i$. (This partial ordering on shapes defines Young's lattice [32, Exercise 3.63]. Additional properties of Young's lattice may be found in various places in [34].) If we relax the conditions that the $\lambda_{i}$ 's are integers but only require them to be real (with $\lambda_{1} \geq \cdots \geq \lambda_{n} \geq 0$ ), then we can think of $\lambda$ as a "fractional shape." Thus $\mathcal{C}_{C}\left(Q_{n}, \boldsymbol{u}\right)$ just consists of the fractional shapes contained in the shape $\left(u_{n}, u_{n-1}, \ldots, u_{1}\right)$.

\section{Connections with Parking Functions and Plane Partitions}

There are two additional interpretations of the volume and lattice point enumerator of $\Pi_{n}(\boldsymbol{x})$ that we wish to discuss. The first concerns the subject of parking functions, originally defined by Konheim and Weiss [9]. A parking function of length $n$ may be defined as a sequence $\left(a_{1}, \ldots, a_{n}\right)$ of positive integers whose increasing rearrangement $b_{1} \leq \cdots \leq b_{n}$ satisfies $b_{i} \leq i$. For the reason for the terminology "parking function," as well as additional results and references, see Exercise 5.49 of [34]. A basic result of Konheim and Weiss is that the number of parking functions of length $n$ is $(n+1)^{n-1}$.

Write park $(n)$ for the set of all parking functions of length $n$. For $\boldsymbol{x}=\left(x_{1}, \ldots, x_{n}\right) \in$ $\mathbb{N}^{n}$ define an $\boldsymbol{x}$-parking function to be a sequence $\left(a_{1}, \ldots, a_{n}\right)$ of positive integers whose increasing rearrangement $b_{1} \leq \cdots \leq b_{n}$ satisfies $b_{i} \leq x_{1}+\cdots+x_{i}$. Thus an ordinary parking function corresponds to the case $\boldsymbol{x}=(1,1, \ldots, 1)$. Let $P_{n}(\boldsymbol{x})$ denote the number of $\boldsymbol{x}$-parking functions. Note that $P_{n}(\boldsymbol{x})=0$ if $x_{1}=0$.

Theorem 11.

$$
P_{n}(\boldsymbol{x})=\sum_{\left(a_{1}, \ldots, a_{n}\right) \in \operatorname{park}(n)} x_{a_{1}} \cdots x_{a_{n}}=n ! V_{n}(\boldsymbol{x})
$$

Proof. Given $\left(a_{1}, \ldots, a_{n}\right) \in \operatorname{park}(n)$, replace each $i$ by an integer in the set $\left\{x_{1}+\cdots+\right.$ $\left.x_{i-1}+1, \ldots, x_{1}+\cdots+x_{i}\right\}$. The number of ways to do this is given by the middle expression in (35), and every $\boldsymbol{x}$-parking function is obtained exactly once in this way. This yields the first equality. The second equality follows from the expansion (2) of 
$V_{n}(\boldsymbol{x})$, since a parking function is obtained by choosing $\boldsymbol{k} \in K_{n}$, forming a sequence with $k_{i} i$ 's, and permuting its elements in $\left(\begin{array}{c}n \\ k_{1}, \ldots, k_{n}\end{array}\right)$ ways.

Take $x_{i}=1$ for all $i$ in (35) and apply (7) for $a=b=1$ to recover the result of [9] that the number of parking functions of length $n$ is $(n+1)^{n-1}$. We note that formula (7) can be given a simple combinatorial proof generalizing the proof of Pollak [5, p. 13] for the case of ordinary parking functions; see p. 10 of [33] for the case $a=b$. We note that Theorem 11 also gives enumerative interpretations of formulae (8) and (9). Presumably these formulae too could be derived combinatorially in the setting of parking functions, but we do not attempt that here.

An interesting special case of Theorem 11 arises when we take $x_{i}=q^{i-1}$ for some $q>0$. In this case we have

$$
n ! V_{n}\left(1, q, q^{2}, \ldots, q^{n-1}\right)=\sum_{\left(a_{1}, \ldots, a_{n}\right) \in \operatorname{park}(n)} q^{a_{1}+\cdots+a_{n}-n} .
$$

It follows from a result of Kreweras [11] (see also Exercise 5.49(c) of [34]) that also

$$
n ! V_{n}\left(1, q, q^{2}, \ldots, q^{n-1}\right)=q^{\left(\begin{array}{c}
n \\
2
\end{array}\right)} I_{n}(1 / q),
$$

where $I_{n}(q)$ is the inversion enumerator of labeled trees.

We can generalize (7) by giving a simple product formula for the Ehrhart polynomial $i\left(\Pi_{n}(\boldsymbol{x}), r\right)$ of $\Pi_{n}(\boldsymbol{x})$ in the case $\boldsymbol{x}=(a, b, b, \ldots, b)$ (see Theorem 13). First we need to discuss another way to interpret $N\left(\Pi_{n}(\boldsymbol{x})\right)$.

Let $\lambda=\left(\lambda_{1}, \ldots, \lambda_{\ell}\right)$ be a partition, so $\lambda_{i} \in \mathbb{N}$ and $\lambda_{1} \geq \cdots \geq \lambda_{\ell} \geq 0$. A plane partition of shape $\lambda$ and largest part at most $m$ is an array $\pi=\left(\pi_{i j}\right)$ of integers $1 \leq \pi_{i j} \leq m$, defined for $1 \leq i \leq \ell$ and $1 \leq j \leq \lambda_{i}$, which is weakly decreasing in rows and columns. For instance, the plane partitions of shape $(2,1)$ and largest part at most 2 are given by

$$
\begin{array}{lllll}
11 & 21 & 22 & 21 & 22 \\
1 & 1 & 1 & 2 & 2
\end{array},
$$

where we only display the positive parts $\pi_{i j}>0$. Basic information on plane partitions may be found in Sections 7.20-7.22 of [34]. If $\boldsymbol{x}=\left(x_{1}, \ldots, x_{n}\right) \in \mathbb{N}^{n}$, then set

$$
\boldsymbol{u}=\left(u_{1}, \ldots, u_{n}\right)=\left(x_{1}, x_{1}+x_{2}, \ldots, x_{1}+\cdots+x_{n}\right)
$$

and write $\tilde{\boldsymbol{u}}=\left(u_{n}, \ldots, u_{1}\right)$, so that $\tilde{\boldsymbol{u}}$ is a partition.

Theorem 12. Let $\boldsymbol{x} \in \mathbb{N}^{n}$. Then $N\left(\Pi_{n}(\boldsymbol{x})\right)$ is equal to the number of plane partitions of shape $\tilde{\boldsymbol{u}}$ and largest part at most 2 .

Proof. If $\left(y_{1}, \ldots, y_{n}\right) \in \Pi_{n}(\boldsymbol{x}) \cap \mathbb{Z}^{n}$, then define the plane partition $\pi$ of shape $\boldsymbol{u}$ to have $y_{1}+\cdots+y_{i}$ twos in row $n+1-i$ and the remaining entries equal to one. This sets up a bijection between the integer points in $\Pi_{n}(x)$ and the plane partitions of shape $\tilde{\boldsymbol{u}}$ and largest part at most 2 . 
Note. Because of the connection given by Theorem 12 between integer points in $\Pi_{n}(x)$ and plane partitions, a number of results concerning $\Pi_{n}(\boldsymbol{x})$ appear already (sometimes implicitly) in the plane partition literature. In particular, consider the determinantal formula (6) of Steck. Let $j_{i}^{\prime}=j_{i}-i, b_{i}^{\prime}=b_{i}-i+1$, and $c_{i}^{\prime}=c_{i}-i-1$. We are then counting sequences $j_{1}^{\prime} \leq j_{2}^{\prime} \leq \cdots \leq j_{n}^{\prime}$ satisfying $b_{i}^{\prime} \leq j_{i}^{\prime} \leq c_{i}^{\prime}$. If $b_{i}^{\prime}>b_{i+1}^{\prime}$, then we can replace $b_{i+1}^{\prime}$ by $b_{i}^{\prime}$ without affecting the sequences $j_{1}^{\prime} \leq \cdots \leq j_{n}^{\prime}$ being counted. Similarly, if $c_{i}^{\prime}>c_{i+1}^{\prime}$ we can replace $c_{i}^{\prime}$ with $c_{i+1}^{\prime}$. Moreover, clearly the number of sequences being counted is not changed by adding a fixed integer $k$ to each $b_{i}^{\prime}$ and $c_{i}^{\prime}$. Hence it costs nothing to assume that $0 \leq b_{1}^{\prime} \leq \cdots \leq b_{n}^{\prime}$ and $0 \leq c_{1}^{\prime} \leq \cdots \leq c_{n}^{\prime}$ (with $\left.b_{i}^{\prime} \leq c_{i}^{\prime}\right)$. Let $\lambda=\left(c_{n}^{\prime}, \ldots, c_{1}^{\prime}\right)$ and $\mu=\left(b_{n}^{\prime}, \ldots, b_{1}^{\prime}\right)$. Then $\lambda$ and $\mu$ are partitions, and $\mu \subseteq \lambda$ in the sense of containment of diagrams (see Section 7.2 of [34]). Let $Y$ denote the poset (actually a distributive lattice) of all partitions of all nonnegative integers, ordered by diagram containment. The lattice $Y$ is just Young's lattice mentioned above. In terms of Young's lattice, we see that that the number \# $(b, c)$ of $(6)$ is just the number of elements $\left(j_{n}^{\prime}, \ldots, j_{1}^{\prime}\right)$ in the interval $[\mu, \lambda]$ of $Y$. Alternatively, $\#(b, c)$ is the number of multichains $\mu=\lambda^{0} \leq \lambda^{1} \leq \lambda^{2}=\lambda$ of length 2 in the interval $[\mu, \lambda]$ of $Y$. Kreweras [10, Section 2.3.7] gives a determinantal formula for the number of multichains of any fixed length $k$ in the interval $[\mu, \lambda]$. (See also Exercise 3.63 of [32].) Such a multichain is easily seen to be equivalent to a plane partition of shape $\lambda / \mu$ with largest part at most $k$. When specialized to $k=2$, Kreweras' formula becomes precisely our (25). Moreover, the special case $\mu=\emptyset$ of Kreweras' formula was already known to MacMahon (put $x=1$ in the implied formula for $G F\left(p_{1} p_{2} \cdots p_{m} ; n\right)[14$, p. 243]). By Theorem 12 the number of elements of the interval $[\emptyset, \lambda]$ is just $N\left(\Pi_{n}(\boldsymbol{x})\right)$, where $\lambda$ is the partition $\tilde{\boldsymbol{u}}$ of Theorem 12. Hence in some sense MacMahon already knew a determinantal formula for $N\left(\Pi_{n}(\boldsymbol{x})\right)$ and thus also (by taking leading coefficients of $N\left(\Pi_{n}(r \boldsymbol{x})\right)$ regarded as a polynomial in $r$ ) for the volume $V_{n}(\boldsymbol{x})$.

Theorem 13. Let $a, b \in \mathbb{N}$ and $\boldsymbol{x}=(a, b, b, \ldots, b) \in \mathbb{N}^{n}$. Then the Ehrhart polynomial $i\left(\Pi_{n}(\boldsymbol{x})\right)$ is given by

$$
i\left(\Pi_{n}(\boldsymbol{x}), r\right)=\frac{1}{n !}(r a+1)(r(a+n b)+2)(r(a+n b)+3) \cdots(r(a+n b)+n) .
$$

In particular, the number $N\left(\Pi_{n}(\boldsymbol{x})\right)$ of integer points in $\Pi_{n}(\boldsymbol{x})$ satisfies

$$
N\left(\Pi_{n}(\boldsymbol{x})\right)=\frac{1}{n !}(a+1)(a+n b+2)(a+n b+3) \cdots(a+n b+n) .
$$

First Proof. The theorem is simply a restatement of a standard result in the subject of ballot problems and lattice path enumeration, going back at least to Lyness [13], and with many proofs. A good discussion appears in Sections 1.4-1.6 of [19]. See also Lemma 3B in Section 1.3 of [20].

Second Proof. We give a proof different from the proofs alluded to above, because it has the virtue of generalizing to give Theorem 14 below. The polytope $r \Pi_{n}(\boldsymbol{x})$ is just $\Pi_{n}(r \boldsymbol{x})$. Hence by Theorem $12 i\left(\Pi_{n}(\boldsymbol{x}), r\right)$ is just the number of plane partitions of shape $\boldsymbol{r} \boldsymbol{u}$ and largest part at most 2. Identify the partition $\boldsymbol{u}$ with its diagram, consisting of all 
pairs $(i, j)$ with $1 \leq i \leq n$ and $1 \leq j \leq \tilde{u}_{i}=a+(n-i) b$. Define the content $c(s)$ of $s=(i, j) \in \tilde{\boldsymbol{u}}$ by $c(s)=j-i$ (see p. 373 of [34]). An explicit formula for the number of plane partitions of shape $\boldsymbol{u}$ and any bound on the largest part was first obtained by Proctor and is discussed in Exercise 7.101 of [34] (as well as a generalization due to Krattenthaler). Proctor's formula for the case at hand gives

$$
i\left(\Pi_{n}(\boldsymbol{x}), r\right)=\prod_{\substack{s=(i, j) \in r \tilde{u} \\ n+c(s) \leq r \tilde{u}_{i}}} \frac{1+n+c(s)}{n+c(s)} \prod_{\substack{s=(i, j) \in \dot{u} \\ n+c(s)>r \tilde{u}_{i}}} \frac{r b+1+n+c(s)}{n+c(s)}
$$

When all the factors of the above products are written out, there is considerable cancellation. The only denominator factors that survive are those indexed by $(i, 1), 1 \leq i \leq n$, yielding the denominator $n$ !. The surviving numerator factors are $r a+1$ (indexed by $(n, r a))$ and $r(a+n b)+k, 2 \leq k \leq n$ (indexed by $(1, r(a+(n-1) b)-n+k))$, the last $n-1$ squares in the first row of $\tilde{\boldsymbol{u}})$.

Note from (36) that the leading coefficient of $i\left(\Pi_{n}(\boldsymbol{x}), r\right)$ (and hence the volume $V_{n}(\boldsymbol{x})$ of $\left.\Pi_{n}(\boldsymbol{x})\right)$ is given by $a(a+n b)^{n-1}$, agreeing with (7).

There is a straightforward generalization of Theorems 12 and 13 involving plane partitions of shape $\boldsymbol{u}$ with largest part at most $m+1$ (instead of just $m+1=2$ ). Given $\boldsymbol{x} \in \mathbb{N}^{n}$ as before, let $\Pi_{n}^{m}(\boldsymbol{x}) \subset \mathbb{R}^{n m}$ be the polytope of all $n \times m$ matrices $\left(y_{i j}\right)$ satisfying $y_{i j} \geq 0$ and

$$
v_{i 1} \leq v_{i 2} \leq \cdots \leq v_{i m} \leq x_{1}+\cdots+x_{i}
$$

for $1 \leq i \leq n$, where

$$
v_{i j}=y_{i 1}+y_{i 2}+\cdots+y_{i j}
$$

Thus $\Pi_{n}^{1}(\boldsymbol{x})=\Pi_{n}(\boldsymbol{x})$. Then the proof of Theorem 12 carries over mutatis mutandis to show that $N\left(\Pi_{n}^{m}(\boldsymbol{x})\right)$ is the number of plane partitions of shape $\tilde{\boldsymbol{u}}$ and largest part at most $m+1$. The result of Proctor mentioned above gives an explicit formula for this number when $\boldsymbol{x}=(a, b, b, \ldots, b)$. Replacing $\boldsymbol{x}$ by $r \boldsymbol{x}$ and computing the leading coefficient of the resulting polynomial in $r$ gives a formula for the volume $V_{n}^{m}(\boldsymbol{x})$ of $\Pi_{n}^{m}(\boldsymbol{x})$. This computation is similar to that in the proof of Theorem 13, though the details are more complicated. We merely state the result here without proof. Is there a direct combinatorial proof similar to the proofs of Theorem 13 (the case $m=1$ of Theorem 14) appearing in [19] and [20]?

Theorem 14. Let $\boldsymbol{x}=(a, b, b, \ldots, b) \in \mathbb{N}^{n}$. Then

$$
(n m) ! V_{n}^{m}(\boldsymbol{x})=1 ! 2 ! \cdots m ! f^{\left\langle m^{n}\right\rangle}(n+m)^{n-1}(n+m-1)^{n-2} \cdots(n+1)^{n-m},
$$

where $f^{\left\langle m^{n}\right\rangle}$ denotes the number of standard Young tableaux of shape $\left\langle m^{n}\right\rangle=(m, m, \ldots$, m) ( $n$ m's in all), given explicitly by the "hook-length formula" [34, Corollary 7.21.6].

\section{A Subdivision of $\Pi_{n}(x)$ Connected with the Associahedron}

In this section we describe a polyhedral subdivison $\left(\hat{\Pi}_{n}(\boldsymbol{k} ; \boldsymbol{x}), \boldsymbol{k} \in K_{n}\right)$ of $\Pi_{n}(\boldsymbol{x})$ different from the subdivision discussed in Section 3. This subdivision is closely related to a 
convex polytope known as the associahedron, defined as follows. Let $E_{n+2}$ be a convex $(n+2)$-gon. A polygonal decomposition of $E_{n+2}$ consists of a set of diagonals of $E_{n+2}$ that do not cross in their interiors. Hence the maximal polygonal decompositions are the triangulations, and contain exactly $n-1$ diagonals. Let $\operatorname{dec}\left(E_{n+2}\right)$ denote the poset of all polygonal decompositions of $E_{n+2}$, ordered by inclusion, with a top element 1 adjoined. It was first shown by Lee [12] and Haiman [7] that $\operatorname{dec}\left(E_{n+2}\right)$ is the face lattice of an $(n-1)$-dimensional convex polytope $\mathcal{A}_{n+2}$, known as the associahedron or Stasheff polytope. (Earlier Stasheff [35] defined the associahedron as a simplicial complex and constructed a geometric realization as a convex body but not as a polytope. Some authors (e.g., p. 18 of [39]) refer to the dual of $\mathcal{A}_{n+2}$ as the associahedron.) A vast generalization is discussed in Chapter 7 of [6]. For some further information see Exercise 6.33 of [34].

We next give a somewhat different description of the associahedron (or, more precisely, of its face lattice) that is most convenient for our purposes. A fan in $\mathbb{R}^{m}$ is a (finite) collection $\boldsymbol{F}$ of pointed polyhedral cones (with vertices at the origin) satisfying the two conditions:

- If $\mathcal{C}, \mathcal{C}^{\prime} \in \boldsymbol{F}$, then $\mathcal{C} \cap \mathcal{C}^{\prime}$ is a face (possibly consisting of just the origin) of $\mathcal{C}$ and $\mathcal{C}^{\prime}$.

- If $\mathcal{C} \in \boldsymbol{F}$ and $\mathcal{C}^{\prime}$ is a face of $\mathcal{C}$, then $\mathcal{C}^{\prime} \in \boldsymbol{F}$.

A fan $\boldsymbol{F}$ is called complete if $\bigcup_{\mathcal{C} \in \boldsymbol{F}}=\mathbb{R}^{m}$.

In analogy to subdivisions of polytopes, the $m$-dimensional cones of a complete fan in $\mathbb{R}^{m}$ are called chambers. We define a fan whose chambers are indexed by plane binary trees with $n$ internal vertices. The definition of a plane tree may be found for instance in the Appendix of [32]. The key point is that the subtrees of any vertex are linearly ordered $T_{1}, \ldots, T_{k}$, indicated in drawing the tree (with the root on the bottom) by placing the subtrees in the order $T_{1}, \ldots, T_{k}$ from left to right. A binary plane tree is a plane tree for which each vertex $v$ has zero or two subtrees. In the latter case we call the vertex an internal vertex. Otherwise $v$ is a leaf or endpoint. We always regard plane trees as being drawn with the root at the bottom.

Let $T$ be a plane binary tree with $n$ internal vertices (so $n+1$ leaves). The number of such trees is the Catalan number $C_{n}$ [34, Exercise 6.19(d)]. Do a depth-first search through $T$ (as defined, e.g., on pp. 33-34 of [34]) and label the internal vertices 1, 2, .,n in the order they are first encountered from above. Equivalently, every internal vertex is greater than those in its left subtree, and smaller than those in its right subtree. We call this labeling of the internal vertices of $T$ the binary search labeling. Figure 7 gives an example when $n=4$. Let $y_{2}, \ldots, y_{n-1}$ denote the coordinates in $\mathbb{R}^{n-1}$. If the internal

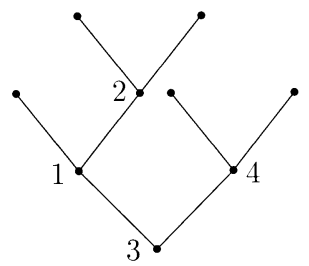

Fig. 7. A plane tree with the binary search labeling of its internal vertices. 
vertex $i$ of $T$ (using the labeling just defined) is the parent of vertex $j$ and $i<j$, then associate with the pair $(i, j)$ the inequality

$$
y_{i+1}+y_{i+2}+\cdots+y_{j} \leq 0,
$$

while if $i>j$, then associate with $(i, j)$ the inequality

$$
y_{j+1}+y_{j+2}+\cdots+y_{i} \geq 0 .
$$

We get a system of $n-1$ homogeneous linear inequalities that define a simplicial cone $\mathcal{C}_{T}$ in $\mathbb{R}^{n-1}$. For example, the inequalities corresponding to the tree of Fig. 7 are given by

$$
\begin{aligned}
y_{2} & \leq 0, \\
y_{2}+y_{3} & \geq 0, \\
y_{4} & \leq 0 .
\end{aligned}
$$

Lemma 15. The $C_{n}$ cones $\mathcal{C}_{T}$, as $T$ ranges over all plane binary trees with $n$ internal vertices, form the chambers of a complete fan $\boldsymbol{F}_{n}$ in $\mathbb{R}^{n-1}$. (For instance, Fig. 8 shows the fan $\boldsymbol{F}_{3}$.)

Proof. Given $1 \leq i \leq n$, let $\mathcal{D}_{i}$ be the cone in $\mathbb{R}^{n-1}$ defined by

$$
\begin{aligned}
y_{2}+y_{3}+\cdots+y_{i} & \geq 0, \\
y_{3}+\cdots+y_{i} & \geq 0,
\end{aligned}
$$

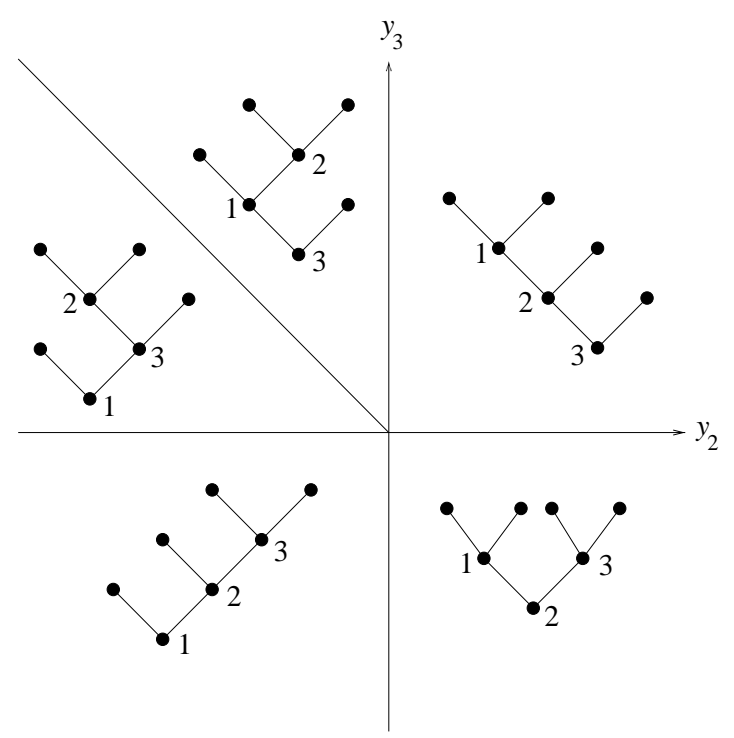

Fig. 8. The fan $\boldsymbol{F}_{3}$. 


$$
\begin{aligned}
y_{i} & \geq 0, \\
y_{i+1} & \leq 0, \\
y_{i+1}+y_{i+2} & \leq 0, \\
& \vdots \\
y_{i+1}+y_{i+2}+\cdots+y_{n} & \leq 0 .
\end{aligned}
$$

Note that

$\mathcal{D}_{i}=\left\{\left(y_{2}, \ldots, y_{n}\right) \in \mathbb{R}^{n-1}: y_{2}+y_{3}+\cdots+y_{i}=\max \left\{y_{2}+y_{3}+\cdots+y_{k}: 1 \leq k \leq n\right\}\right\}$.

In particular,

$$
\mathcal{D}_{1}=\left\{\left(y_{2}, \ldots, y_{n}\right) \in \mathbb{R}^{n-1}: y_{2}+y_{3}+\cdots+y_{k} \leq 0,2 \leq k \leq n\right\} .
$$

Claim. Let $\mathcal{T}_{i}$ consist of all plane binary trees with $n$ internal vertices and with root $i$ (in the binary search labeling). Then

$$
\mathcal{D}_{i}=\bigcup_{T \in \mathcal{T}_{i}} \mathcal{C}_{T}
$$

The proof of the claim is by induction on $n$, the cases $n=1$ and $n=2$ being trivial to check. Let $n \geq 3$, and assume the claim for all $m<n$. Let $T \in \mathcal{T}_{i}$. Hence by the induction hypothesis, the set of all possible left subtrees $T_{1}$ with root $j$ of the root $i$ of $T$ defines all points $\left(y_{2}, y_{3}, \ldots, y_{i-1}\right) \in \mathbb{R}^{i-1}$ such that $y_{2}+y_{3}+\cdots+y_{j}$ is the maximum partial sum of the sequence $\left(y_{2}, \ldots, y_{i-1}\right)$. Since generically vertex $j$ will be the left child of the root $i$ (because the maximum partial sum $y_{2}+y_{3}+\cdots+y_{k}$ will occur for a unique $k$ ), we obtain the additional inequality $y_{j+1}+y_{j+2}+\cdots+y_{i} \geq 0$. This means that $y_{2}+y_{3}+\cdots+y_{i}$ is the maximum partial sum of the sequence $\left(y_{2}, y_{3}, \ldots, y_{i}\right)$. Similarly, the set of all possible right subtrees $T_{2}$ with root $j$ of the root $i$ of $T$ defines all points $\left(y_{i+1}, y_{i+2}, \ldots, y_{n}\right) \in \mathbb{R}^{n-i}$ such that $y_{i+1}+y_{i+2}+\cdots+y_{j}$ is the maximum partial sum of the sequence $\left(y_{i+1}, y_{i+2}, \ldots, y_{n}\right)$. Since generically $j$ will be a child of the root $i$, we obtain the additional inequality $y_{i+1}+y_{i+2}+\cdots+y_{j} \leq 0$. This means that

$$
y_{2}+y_{3}+\cdots+y_{k} \leq y_{2}+y_{3}+\cdots+y_{i}, \quad \text { for all } i+1 \leq k \leq n .
$$

Hence $\mathcal{D}_{i}=\bigcup_{T \in \mathcal{T}_{i}} \mathcal{C}_{T}$, so the proof of the claim follows by induction.

From the definition of $\mathcal{D}_{i}$ it is clear that

$$
\bigcup_{i=1}^{n} \mathcal{D}_{i}=\mathbb{R}^{n-1}
$$

The proof of the lemma then follows from (39) and (40). 


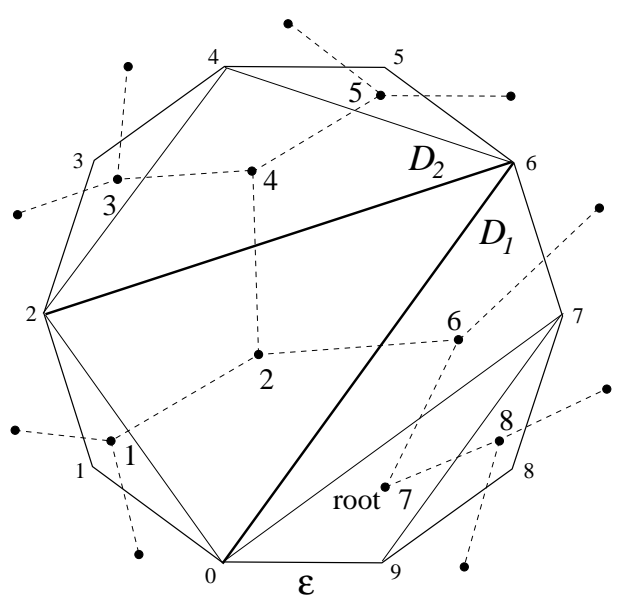

Fig. 9. A triangulated 10-gon and the corresponding plane binary tree $T$.

Theorem 16. The face poset $P\left(\boldsymbol{F}_{n}\right)$ of the fan $\boldsymbol{F}_{n}$, with a top element $\hat{1}$ adjoined, is isomorphic to the face lattice dec $\left(E_{n+2}\right)$ of the associahedron $\mathcal{A}_{n+2}$.

Proof. The face lattice of a complete fan is completely determined by the incidences between the chambers and rays (one-dimensional faces). (See Exercise 3.12 of [32] for a stronger statement.) The chambers of $\boldsymbol{F}_{n}$ (proved to be a complete fan in Lemma 15) have already been described in terms of plane binary trees. There is a well-known bijection between plane binary trees on $2 n+1$ vertices and triangulations of a convex $(n+2)$-gon $E_{n+2}$. This bijection is explained for instance in Corollary 6.2.3 of [34]. In particular, to define the bijection we first need to fix an edge $\varepsilon$ of $E_{n+2}$, called the root edge. We hope that Fig. 9 will make this bijection clear; see the previous reference for further details. Thus we have a bijection between the chambers $\mathcal{C}$ of $\boldsymbol{F}_{n}$ and the triangulations of the convex $(n+2)$-gon $E_{n+2}$.

We now describe the rays $R$ of $\boldsymbol{F}_{n}$. We can describe $R$ uniquely by specifying one nonzero point on $R$. We index these points by the diagonals $D$ of a convex $(n+2)$-gon $E_{n+2}$. Label the vertices of $E_{n+2}$ as $0,1, \ldots, n+1$ clockwise beginning with one vertex of $\varepsilon$ and ending with the other. Let $e_{i}$ denote the unit coordinate vector corresponding to the coordinate $y_{i}$ in the space $\mathbb{R}^{n-1}$ with coordinates $y_{2}, \ldots, y_{n}$. Given the diagonal $D$ between vertices $i<j$ of $E_{n+2}$, associate a point $p_{D} \in \mathbb{R}^{n-1}$ as follows:

$$
p_{D}= \begin{cases}e_{j}, & \text { if } i=0, \\ -e_{i+1}, & \text { if } j=n+1, \\ e_{j}-e_{i+1}, & \text { otherwise. }\end{cases}
$$

We claim that the ray $\left\{\alpha p_{D}: \alpha \in \mathbb{R}_{\geq 0}\right\}$ is the ray of $\boldsymbol{F}_{n}$ that is the intersection of all the chambers of $\boldsymbol{F}_{n}$ corresponding to the triangulations of $E_{n+2}$ that contain $D$. From this claim the proof of the theorem follows (using the fact that $\boldsymbol{F}_{n}$ is a simplicial fan, i.e., every face is a simplicial cone). 
Consider first the diagonal $D$ with vertices 0 and $j$. Let $\Upsilon$ be a triangulation of $E_{n+2}$ containing $D$. The internal vertices of $T$ correspond to the regions (triangles) of the triangulation $\Upsilon$. Because of our procedure for labeling the internal vertices of a plane binary tree $T$, it follows that the labels of the internal vertices "above" $D$ (i.e., on the opposite side of $D$ as the root edge $\varepsilon$ ) will be $1,2, \ldots, j-1$, while the internal vertices below $D$ will be labeled $j, j+1, \ldots, n$. (See Fig. 9 for an example with $n=8$. The diagonal $D$ in question is labeled $D_{1}$ and connects vertex 0 to vertex $j=6$. The plane binary tree $T$ is drawn with dashed lines.) Consider the internal edges of $T$ that give rise (via (37) and (38)) to chambers whose equations involve $y_{j}$. No such edge can appear below $D$, since $j$ is the least vertex label appearing below $D$. Similarly no such edge can appear above $D$, since only vertices less than $j$ appear above $D$. Hence such an edge must cross $D$. The top (farthest from the root) vertex $a$ of this edge is $<j$, while the bottom vertex $b$ is $\geq j$. Hence the chamber equation is given by $y_{a+1}+y_{a+2}+\cdots+y_{b} \geq 0$, where $a<j$ and $b \geq j$. Hence the point $e_{j}$ lies on this chamber, and so the ray through $e_{j}$ is the intersection of the chambers corresponding to triangulations containing $D$.

A completely analogous argument holds for the diagonal $D$ with vertices $i$ and $n+1$.

Finally suppose that $D$ has vertices $i, j$ where $0<i<j<n+1$. The internal vertices of $T$ appearing above $D$ will be labeled $i+1, i+2, \ldots, j-1$, while the remaining vertex labels appear below $D$. (See Fig. 9, where the diagonal $D$ in question is labeled $D_{2}$, and where $i=2$ and $j=6$.) Consider an internal edge of $T$ whose vertex labels are $a$ and $b$ where $a \leq i$ and $i+1 \leq b<j$. These are precisely the edges whose corresponding chamber equation (either $y_{a+1}+y_{a+2}+\cdots+y_{b} \geq 0$ or $y_{a+1}+y_{a+2}+\cdots+y_{b} \leq 0$ ) involves $y_{i+1}$ but not $y_{j}$. Since $b$ appears above $D$ and $a$ below, the chamber equation is in fact $y_{a+1}+y_{a+2}+\cdots+y_{b} \leq 0$. In particular, the point $e_{j}-e_{i+1}$ lies on the chamber. Similarly, consider an internal edge of $T$ whose labels are $a$ and $b$ where $i+1 \leq a<j$ and $j \leq b$. These are precisely the edges whose corresponding chamber equation (again either $y_{a+1}+y_{a+2}+\cdots+y_{b} \geq 0$ or $y_{a+1}+y_{a+2}+\cdots+y_{b} \leq 0$ ) involves $y_{j}$ but not $y_{i+1}$. Since $b$ appears below $D$ and $a$ above, the chamber equation is in fact $y_{a+1}+y_{a+2}+\cdots+y_{b} \geq 0$. In particular, the point $e_{j}-e_{i+1}$ lies on the chamber. Every other chamber equation either involves both $y_{i+1}$ and $y_{j}$ (with a coefficient 1), or else involves neither. Hence $e_{i+1}-e_{j}$ lies on every chamber corresponding to a triangulation containing $D$, so the intersection of these chambers is the ray containing $e_{j}-e_{i+1}$. This completes the proof of the claim, and with it the theorem.

The connection between $\Pi_{n}(\boldsymbol{x})$ and the fan $\boldsymbol{F}_{n}$ is provided by the concept of a plane tree with edge lengths. If we associate with each edge $e$ of the plane tree $T$ a positive real number $\ell(e)$, then we call the pair $(T, \ell)$ a plane tree with edge lengths. Such a tree can be drawn by letting the length of each edge $e$ be $\ell(e)$.

Now fix a real number $s>0$, which will be the sum of the edge lengths of a plane tree. Let $\boldsymbol{x}=\left(x_{1}, \ldots, x_{n}\right) \in \mathbb{R}_{+}^{n}$ with $\sum x_{i}<s$. Let $\boldsymbol{y}=\left(y_{1}, \ldots, y_{n}\right) \in \mathbb{R}_{+}^{n}$ with $y_{1}+\cdots+y_{i} \leq x_{1}+\cdots+x_{i}$ for $1 \leq i \leq n$. We associate with the pair $(\boldsymbol{x}, \boldsymbol{y})$ a plane tree with edge lengths $\varphi(x, y)=(\bar{T}, \ell)$ as follows. Start at the root and traverse the tree in preorder (or depth-first order) [34, pp. 33-34]. First go up a distance $x_{1}$, then down a distance $y_{1}$, then up a distance $x_{2}$, then down a distance $y_{2}$, etc. After going down a distance $y_{n}$, complete the tree by going up a distance $x_{n+1}=s-x_{1}-\cdots-x_{n}$ and then 


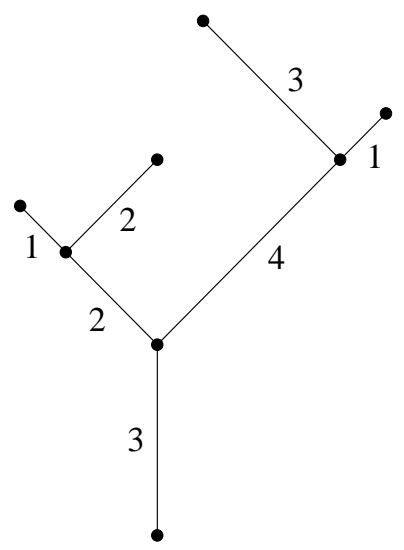

Fig. 10. A planted plane binary tree with edge lengths.

down a distance $y_{n+1}=s-y_{1}-\cdots-y_{n}$. Generically we obtain a planted plane binary tree with edge lengths, i.e, the root has degree 1 (or one child), and all other internal vertices have degree 2 . Figure 10 shows the planted plane binary tree with edge lengths associated with $s=16$ and $\boldsymbol{x}=(6,2,7), \boldsymbol{y}=(1,4,3)$. If $\bar{T}$ is a planted plane tree, then we let $T$ denote the tree obtained by "unplanting" (uprooting?) $\bar{T}$, i.e., remove from $\bar{T}$ the root and its unique incident edge $e$ (letting the other vertex of $e$ become the root of $T$ ).

Fix the sequence $\boldsymbol{x}=\left(x_{1}, \ldots, x_{n}\right)$ with $\sum x_{i}<s$. For a plane binary tree $T$ (without edge lengths) with $n$ internal vertices (and hence $n+1$ leaves), define $\Delta_{T}=\Delta_{T}(\boldsymbol{x})$ to be the set of all $\boldsymbol{y}=\left(y_{1}, \ldots, y_{n}\right) \in \mathbb{R}_{+}^{n}$ such that $\varphi(\boldsymbol{x}, \boldsymbol{y})=(\bar{T}, \ell)$ for some $\ell$. Let $\mathcal{T}_{n}$ denote the set of plane binary trees with $n$ internal vertices. Let $T \in \mathcal{T}_{n}$ with the binary search labeling of its internal vertices as defined earlier in this section. We now define a sequence $\boldsymbol{k}(T)=\left(k_{1}, \ldots, k_{n}\right) \in \mathbb{N}^{n}$ as follows: (1) $k_{i}=0$ if the left child of vertex $i$ is an internal vertex. (2) If the left child of vertex $i$ is an endpoint, then let $k_{i}$ be the largest integer $r$ for which there is a chain $i=j_{1}<j_{2}<\cdots<j_{r}$ of internal vertices such that $j_{h}$ is a left child of $j_{h+1}$ for $1 \leq h \leq r-1$. For instance, if $T$ is the tree of Fig. 11, then $\boldsymbol{k}(T)=(2,3,0,1,0,1,0,2,0)$.

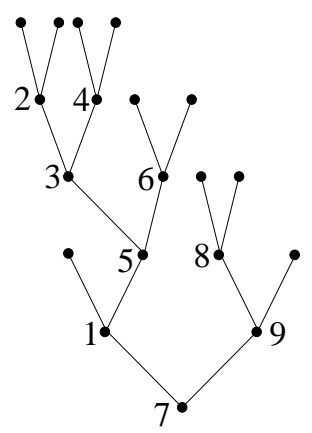

Fig. 11. A plane binary tree $T$ with $\boldsymbol{k}(T)=(2,3,0,1,0,1,0,2,0)$. 
Lemma 17. The map $T \mapsto \boldsymbol{k}(T)$ is a bijection from $\mathcal{T}_{n}$ to the set $K_{n}$ defined by (3).

Proof. Let $\boldsymbol{k}(T)=\left(k_{1}, \ldots, k_{n}\right)$. The chains $i=j_{1}<j_{2}<\cdots<j_{r}$ described above partition the internal vertices of $T$, so $\sum k_{i}=n$. Since $k_{j_{2}}=\cdots=k_{j_{r}}=0$, it follows that $k_{h+1}+k_{h+2}+\cdots+k_{n} \leq n-h$ for $0 \leq h \leq n-1$. Hence $k_{1}+\cdots+k_{h} \geq h$, so $\boldsymbol{k}(T) \in K_{n}$.

It remains to show that given $\boldsymbol{k}=\left(k_{1}, \ldots, k_{n}\right) \in K_{n}$, there is a unique $T \in \mathcal{T}_{n}$ such that $\boldsymbol{k}(T)=\boldsymbol{k}$. We can construct the subtree of internal vertices of $T$ as follows. Let $T_{1}$ be defined by starting at the root and making $k_{1}-1$ steps to the left. (Each step is from a vertex to an adjacent vertex.) Hence we have $k_{1}$ vertices in all, and we are located at the vertex furthest from the root. Suppose that $T_{i}$ has been constructed for $i<n$, and that we are located at vertex $v_{i}$. If $k_{i+1}>0$, then move one step to the right and $k_{i+1}-1$ steps to the left, yielding the tree $T_{i+1}$ and the vertex $v_{i+1}$ at which we are located. If $k_{i+1}=0$, then move down the tree (toward the root) until we have traversed exactly one edge in a southeast direction. This gives the tree $T_{i+1}=T_{i}$ and a new present location $v_{i+1}$. Let $T=T_{n}$. It is easily checked that the definition of $K_{n}$ ensures that $T$ is defined (and, though not really needed here, that $v_{n}$ is the root vertex) and $\boldsymbol{k}(T)=\boldsymbol{k}$. Since there are $C_{n}=(1 /(n+1))\left(\begin{array}{c}2 n \\ n\end{array}\right)$ plane binary trees with $n$ internal vertices and since $\# K_{n}=C_{n}$, it follows that the map $T \mapsto \boldsymbol{k}(T)$ is a bijection as claimed. (It is also easy to see directly that $T$ is unique, i.e., if $\boldsymbol{k}(T)=\boldsymbol{k}\left(T^{\prime}\right)$, then $T=T^{\prime}$.)

Now given $t \in \mathbb{R}_{+}$, let $\sigma_{k}(t)$ denote the $k$-dimensional simplex of points $\left(t_{1}, \ldots, t_{k}\right)$ satisfying $0 \leq t_{1} \leq t_{2} \leq \cdots \leq t_{k} \leq t$. Thus

$$
\operatorname{Vol}\left(\sigma_{k}(t)\right)=\frac{t^{k}}{k !}
$$

By convention $\sigma_{0}(t)$ is just a point, with $\operatorname{Vol}\left(\sigma_{0}(t)\right)=1$. Define two compact subsets $\mathcal{X}$ and $\mathcal{Y}$ of $\mathbb{R}^{n}$ to be unimodularly equivalent if there is an affine transformation of determinant \pm 1 that maps $\mathcal{X}$ onto $\mathcal{Y}$. $(\operatorname{Hence} \operatorname{Vol}(\mathcal{X})=\operatorname{Vol}(\mathcal{Y})$.) We can now state the main result of this section.

Theorem 18. (a) The sets $\Delta_{T}(\boldsymbol{x})$, for $T \in \mathcal{T}_{n}$, form the maximal faces (chambers) of a polyhedral decomposition $\Gamma_{n}$ of $\Pi_{n}(\boldsymbol{x})$.

(b) Let $\boldsymbol{k}(T)=\left(k_{1}, \ldots, k_{n}\right)$, where $T \in \mathcal{T}_{n}$. Then $\Delta_{T}(\boldsymbol{x})$ is unimodularly equivalent to the product $\sigma_{k_{1}}\left(x_{1}\right) \times \cdots \times \sigma_{k_{n}}\left(x_{n}\right)$, so in particular

$$
\operatorname{Vol}\left(\Delta_{T}(\boldsymbol{x})\right)=\frac{x_{1}^{k_{1}}}{k_{1} !} \cdots \frac{x_{n}^{k_{n}}}{k_{n} !}
$$

(c) The interior face complex $\Gamma_{n}^{\circ}$ of $\Gamma_{n}$ is combinatorially equivalent to the associahedron, i.e., the set of interior faces of $\Gamma_{n}$, ordered by inclusion, is isomorphic to the face lattice of the associahedron.

Proof. (a) The construction of the plane tree with edge lengths $\varphi(\boldsymbol{x}, \boldsymbol{y})=(\bar{T}, \ell)$ is defined if and only if $\boldsymbol{y} \in \Pi_{n}(\boldsymbol{x})$. Since generically $\varphi(\boldsymbol{x}, \boldsymbol{y})$ is a planted plane binary tree, 
it follows that the sets $\Delta_{T}(\boldsymbol{x}), T \in \mathcal{T}_{n}$, form the chambers of a polyhedral decomposition of $\Pi_{n}(\boldsymbol{x})$.

(b) Let $\varphi(\boldsymbol{x}, \boldsymbol{y})=(\bar{T}, \ell)$ as above. Call a vertex $v$ of $\bar{T}$ a left leaf if it is a leaf (endpoint) and is the left child of its parent. Similarly a right edge is an edge that slants to the right as we move away from the root. Let $P(v)$ be the path from the left leaf $v$ toward the root that terminates after the first right edge is traversed (or terminates at the root if there is no such right edge). Let $c(v)$ be the label of the (internal) vertex that is the parent of $v$. Then the length of the path $P(v)$ is just $x_{c(v)}$. If $c(v)=i$, then exactly $k_{i}$ of the paths $P(u)$ end at the path $P(v)$. Suppose that these paths are $P\left(u_{1}\right), \ldots, P\left(u_{k_{i}}\right)$ where $u_{1}<\cdots<u_{k_{i}}$. Then the paths $P\left(u_{j}\right)$ intersect the path $P(v)$ in the order $P\left(u_{1}\right), \ldots, P\left(u_{k_{i}}\right)$ from the bottom up. Hence for each $i$ with $k_{i}>0$, we can independently place on a path of length $x_{i}$ the $k_{i}$ points that form the bottoms of the paths $P\left(u_{j}\right)$. The placement of these points defines a point in a simplex unimodularly equivalent to $\sigma_{k_{i}}\left(x_{i}\right)$, so $\Delta_{T}(\boldsymbol{x})$ is unimodularly equivalent to $\sigma_{k_{1}}\left(x_{1}\right) \times \cdots \times \sigma_{k_{n}}\left(x_{n}\right)$ as claimed.

Example 19. Let $\bar{T}$ be the planted plane binary tree of Fig. 12. On the path of length $x_{1}$ from the root $r$ to $v_{1}$ we can place vertices 1 and 3 in bijection with the points of the simplex $0 \leq t_{3} \leq t_{1} \leq x_{1}$ of volume $x_{1}^{2} / 2$. On the path of length $x_{2}$ from 1 to $v_{2}$ we can place vertex 2 in bijection with the points of the simplex $0 \leq t_{2} \leq x_{2}$, of volume $x_{2}$. Finally on the path of length $x_{4}$ from 3 to $v_{3}$ we can place vertices $4,5,6$ in bijection with the points of the simplex $0 \leq t_{6} \leq t_{5} \leq t_{4} \leq x_{4}$, of volume $x_{4}^{3} / 6$. Hence $\Delta_{T}$ is unimodularly equivalent to the product $\sigma_{2}\left(x_{1}\right) \times \sigma_{1}\left(x_{2}\right) \times \sigma_{3}\left(x_{4}\right)$, of volume $x_{1}^{2} x_{2} x_{4}^{3} / 2$ ! 1 ! 3 !.

It is easy to make the unimodular equivalence between $\Delta_{T}$ and $\sigma_{k_{1}}\left(x_{1}\right) \times \cdots \times \sigma_{k_{n}}\left(x_{n}\right)$ completely explicit. For instance, in the above example $t_{3}$ is the distance between vertices $r$ and 3 , so

$$
t_{3}=x_{1}-y_{1}+x_{2}-y_{2}+x_{3}-y_{3} .
$$

Similarly,

$$
t_{1}=x_{1}-y_{1}
$$

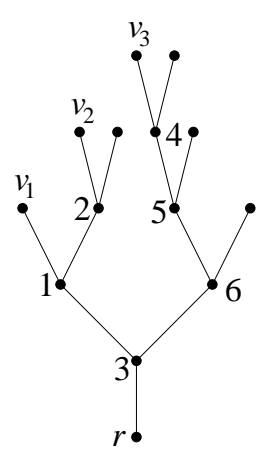

Fig. 12. A planted plane binary tree. 
Now $t_{2}$ is the distance between vertices 1 and 2 , so

$$
t_{2}=x_{2}-y_{2}
$$

In the same way we obtain

$$
\begin{aligned}
& t_{6}=x_{4}-y_{4}+x_{5}-y_{5}+x_{6}-y_{6}, \\
& t_{5}=x_{4}-y_{4}+x_{5}-y_{5}, \\
& t_{4}=x_{4}-y_{4} .
\end{aligned}
$$

Proof of Theorem 18(c). Let $\varphi(\boldsymbol{x}, \boldsymbol{y})=(\bar{T}, \ell)$. Then the height (or distance from the root) of vertex $i$ is just $x_{1}+\cdots+x_{i}-y_{1}-\cdots-y_{i}=u_{i}-v_{i}$. Hence if vertex $i$ is the parent of $j$, then $u_{i}-v_{i}<u_{j}-v_{j}$. If $i<j$ we get the equation

$$
\left(y_{i+1}-x_{i+1}\right)+\cdots+\left(y_{j}-x_{j}\right) \leq 0,
$$

while if $i>j$ we get

$$
\left(y_{j+1}-x_{j+1}\right)+\cdots+\left(y_{i}-x_{i}\right) \geq 0 .
$$

Thus these $n-1$ equations, together with $y_{i} \geq 0$ and $y_{1}+\cdots+y_{i} \leq x_{1}+\cdots+x_{i}$, determine $\bar{\Delta}_{T}$.

Note that if we replace each $y_{k}$ by $y_{k}-x_{k}$ in the inequalities (37) and (38) defining the chambers of the fan $\boldsymbol{F}_{n}$ of Theorem 16, then we obtain precisely the inequalities (41) and (42). From this we conclude the following. Given $\boldsymbol{x}=\left(x_{1}, \ldots, x_{n}\right) \in \mathbb{R}_{\geq 0}^{n}$, translate the fan $\boldsymbol{F}_{n}$ so that the center of the translated fan $\tilde{\boldsymbol{F}}_{n}$ is at $\left(x_{2}, \ldots, x_{n}\right)$. Add a new $y_{1}$ axis and lift $\tilde{\boldsymbol{F}}_{n}$ into $\mathbb{R}^{n}$, giving a "nonpointed fan" (i.e., a decomposition of $\mathbb{R}^{n}$ satisfying the definition of a fan except that the cones are nonpointed) which we denote by $\mathbb{R} \times \tilde{\boldsymbol{F}}_{n}$. (Thus each cone $\mathcal{C} \in \tilde{\boldsymbol{F}}_{n}$ lifts to the nonpointed cone $\mathbb{R} \times \mathcal{C}$.) Finally intersect each chamber (maximal cone) $\mathbb{R} \times \mathcal{C}$ of $\mathbb{R} \times \tilde{\boldsymbol{F}}_{n}$ with the polytope $\Pi_{n}(\boldsymbol{x})$. Then the polytopes $\mathcal{C} \cap \Pi_{n}(\boldsymbol{x})$ are just the chambers $\hat{\Pi}(\boldsymbol{k} ; \boldsymbol{x})$ of the polyhedral decomposition $\mathcal{P}_{n}$ of $\Pi_{n}(\boldsymbol{x})$. Moreover, the interior faces of this decomposition are just the intersections of arbitrary cones in $\mathbb{R} \times \tilde{\boldsymbol{F}}_{n}$ with $\Pi_{n}(\boldsymbol{x})$. Hence the interior face poset of $\mathcal{P}_{n}$ is isomorphic to the face poset of the fan $\boldsymbol{F}_{n}$, which by Theorem 16 is the face lattice of the associahedron.

Notes. The decomposition of $\Pi_{n}(\boldsymbol{x})$ given by Theorem 16 is fundamentally different (i.e., has a different combinatorial type) than that of Theorem 8. For instance, when $n=3$ Fig. 6 shows that the interior face dual complex described by Theorem 8 is not a decomposition of a convex polytope, unlike the situation in Theorem 16. In that case when $n=3$ the interior face dual complex is just a solid pentagon. The two subdivisions of $\Pi_{3}(\boldsymbol{x})$ are shown explicitly in Fig. 2.

We are grateful to Victor Reiner for pointing out to us that Theorem 16 is related to the construction of the associahedron appearing in [12] and [26], and that a $B_{n}$-analogue of this construction appears in Section 3 of [1]. Note that the proof of Theorem 16 shows that the rays of the fan $\boldsymbol{F}_{n}$ are the vectors $e_{i}$ and $-e_{i}$ for $1 \leq i \leq n-1$, and $e_{i}-e_{j}$ for $1 \leq i<j \leq n-1$. As pointed out to us by Reiner, it follows from [12] that we 
can rescale these vectors (i.e., multiply them by suitable positive real numbers) so that their convex hull is combinatorially equivalent (as defined in the next section) to the associahedron $\mathcal{A}_{n+2}$.

Some of the results of this section can be interpreted probabilistically in terms of the kind of random plane tree with edge lengths derived from a Brownian excursion by Neveu and Pitman [21]. It was in fact by consideration of such random trees that we were first led to the formula (2) for the volume polynomial, with the geometric interpretation provided by Theorem 18 .

\section{The Face Structure of $\Pi_{n}(x)$}

In this section we determine the structure of the faces of $\Pi_{n}(\boldsymbol{x})$, i.e., a description of the lattice of faces of $\Pi_{n}(\boldsymbol{x})$ (ordered by inclusion). This description will depend on the "degeneracy" of $\Pi_{n}(\boldsymbol{x})$, i.e., for which $i$ we have $x_{i}=0$. Thus let $u_{i}=x_{1}+\cdots+x_{i}$ as usual, and define integers $1 \leq a_{1}<a_{2}<\cdots<a_{k}=n$ by

$$
u_{1}=\cdots=u_{a_{1}}<u_{a_{1}+1}=\cdots=u_{a_{2}}<\cdots<u_{a_{k-1}+1}=\cdots=u_{a_{k}} .
$$

We say that two convex polytopes are combinatorially equivalent or have the same combinatorial type if they have isomorphic face lattices.

Theorem 20. Let $a_{1}, \ldots, a_{k}$ be as above, and set $b_{i}=a_{i}-a_{i-1}$ (with $a_{0}=0$ ). Assume (without loss of generality) that $x_{1}>0$. Then $\Pi_{n}(\boldsymbol{x})$ is combinatorially equivalent to a product $\sigma_{b_{1}} \times \cdots \times \sigma_{b_{k}}$, where $\sigma_{j}$ denotes a $j$-simplex. In particular, if each $x_{i}>0$ then $\Pi_{n}(\boldsymbol{x})$ is combinatorially equivalent to an $n$-cube.

Proof. For $1 \leq i \leq k$, let $\mathcal{S}_{i}=\left\{C_{i 0}, C_{i 1}, \ldots, C_{i, b_{i}}\right\}$ denote the set of the following $b_{i}+1$ conditions $C_{i j}$ on a point $y \in \Pi_{n}(\boldsymbol{x})$ :

$$
\begin{aligned}
& \left(C_{i 0}\right) \quad y_{a_{i-1}+1}=y_{a_{i-1}+2}=\cdots=y_{a_{i}}=0, \\
& \left(C_{i 1}\right) \quad y_{a_{i-1}+1}=u_{i}, \quad y_{a_{i-1}+2}=y_{a_{i-1}+3}=\cdots=y_{a_{i}}=0 \text {, } \\
& \left(C_{i 2}\right) \quad y_{a_{i-1}+2}=u_{i}, \quad y_{a_{i-1}+1}=y_{a_{i-1}+3}=\cdots=y_{a_{i}}=0 \text {, } \\
& \left(C_{i, b_{i}}\right) \quad y_{a_{i}}=u_{i}, \quad y_{a_{i-1}+1}=y_{a_{i-1}+2}=\cdots=y_{a_{i}-1}=0 .
\end{aligned}
$$

Note that each of the conditions $C_{i j}$ consists of $b_{i}$ chambers of $\Pi_{n}(\boldsymbol{x})$; we regard $C_{i j}$ as being the set of these chambers. Let $S_{i}$ denote any subset of $\mathcal{S}_{i}$, and let $\bigcap S_{i}=\bigcap_{C \in S_{i}} C$. A little thought shows that we can find a point $y \in \Pi_{n}(\boldsymbol{x})$ lying on all the chambers in each $\bigcap S_{i}$, but not lying on any other chamber of $\Pi_{n}(\boldsymbol{x})$. Moreover, no point of $\Pi_{n}(\boldsymbol{x})$ can lie on any other collection of chambers of $\Pi_{n}(x)$ but on no additional chambers.

From the above discussion it follows that $\Pi_{n}(\boldsymbol{x})$ is combinatorially equivalent to a product of simplices of dimensions $b_{1}, \ldots, b_{k}$, as desired. In particular, $\Pi_{n}(\boldsymbol{x})$ has $\left(b_{1}+1\right)\left(b_{2}+1\right) \cdots\left(b_{k}+1\right)$ vertices $v$, obtained by choosing $0 \leq j_{i} \leq b_{i}$ for each $i$ and defining $v$ to be the intersection of the chambers in all the $C_{i j_{i}}$ 's. 
Although $\Pi_{n}(x)$ is combinatorial equivalent to a product of simplices, it is not the case that $\Pi_{n}(\boldsymbol{x})$ is affinely equivalent to such a product. For instance, Fig. 1 shows $\Pi_{2}\left(x_{1}, x_{2}\right)$ when $x_{1}, x_{2}>0$. We see that $\Pi_{2}\left(x_{1}, x_{2}\right)$ is a quadrilateral and hence combinatorially equivalent to a square. However, $\Pi_{2}\left(x_{1}, x_{2}\right)$ is not a parallelogram and hence not affinely equivalent to a square. Similarly Fig. 2 shows that $\Pi_{3}\left(x_{1}, x_{2}, x_{3}\right)$ is combinatorially equivalent but not affinely equivalent to a 3 -cube when each $x_{i}>0$.

\section{References}

1. H. Burgiel and V. Reiner, Two signed associahedra, New York J. Math. (electronic) 4 (1998), 83-95.

2. E. Csáki and G. Tusnády, On the number of intersections and the ballot theorem, Period. Math. Hungar. 2 (1972), 5-13

3. H. E. Daniels, The statistical theory of the strength of bundles of thread, Proc. Roy. Soc. London Ser. A 183 (1945), 405-435.

4. W. Feller, An Introduction to Probability Theory and Its Applications, Vol. 2, Wiley, New York, 1966.

5. D. Foata and J. Riordan, Mappings of acyclic and parking functions, Aequationes Math 10 (1974), 10-22.

6. I. M. Gelfand, M. M. Kapranov, and A. V. Zelevinsky, Discriminants, Resultants, and Multidimensional Determinants, Birkhäuser, Boston, 1994.

7. M. Haiman, Constructing the associahedron, Unpublished handwritten manuscript.

8. N. L. Johnson, S. Kotz, and N. Balakrishnan, Discrete Multivariate Distributions, Wiley, New York, 1997.

9. A. G. Konheim and B. Weiss, An occupancy discipline and applications, SIAM J. Appl. Math. 14 (1966), $1266-1274$.

10. G. Kreweras, Sur une classe de problèmes de dénombrement liès au treillis des partitions des entiers, Cahiers du BURO, no. 6, Institut de Statistique de L'Univ. Paris, 1965.

11. G. Kreweras, Une famille de polynômes ayant plusieurs propriétés énumeratives, Period. Math. Hungar. 11 (1980), 309-320.

12. C. W. Lee, The associahedron and triangulations of the $n$-gon, European J. Combin. 10 (1989), 551-560.

13. R. C. Lyness, Al Capone and the death ray, Math. Gaz. 25 (1941), 283-287.

14. P. A. MacMahon, Combinatory Analysis, Vols. 1 and 2, Chelsea, New York, 1960.

15. J. F. Marckert and Ph. Chassaing, Parking functions, empirical processes, and the width of rooted labeled trees, Preprint available via http://www.iecn.u-nancy.fr/ chassain//theme.html, 1999.

16. P. McMullen, Valuations and Euler-type relations on certain classes of convex polytopes. Proc. London Math. Soc. (3) 35 (1977), 113-135.

17. P. McMullen, Valuations and dissections, in Handbook of Convex Geometry (P. M. Gruber and J. M. Wills, eds.), Vols. A and B, pp. 933-988, North-Holland, Amsterdam, 1993.

18. P. McMullen and R. Schneider, Valuations on convex bodies, in Convexity and Its Applications (P. M. Gruber and J. M. Wills, eds.), pp. 170-247, Birkhäuser, Basel, 1983.

19. S. G. Mohanty, Lattice Path Counting and Applications, Academic Press, New York, 1979.

20. T. V. Narayana, Lattice Path Combinatorics with Statistical Applications, Mathematical Expositions No. 23, University of Toronto Press, Toronto, 1979.

21. J. Neveu and J. Pitman, Renewal property of the extrema and tree property of a one-dimensional Brownian motion, in Séminaire de Probabilités XXIII, pp. 239-247, Lecture Notes in Mathematics, Vol. 1372, Springer-Verlag, Berlin, 1989.

22. H. Niederhausen, Sheffer polynomials for computing exact Kolmogorov-Smirnov and Rényi type distributions, Ann. Statist. 9 (1981), 923-944.

23. E. J. G. Pitman, Simple proofs of Steck's determinantal expressions for probabilities in the Kolmogorov and Smirnov tests, Bull. Austral. Math. Soc. 7 (1972), 227-232.

24. E. J. G. Pitman, Some Basic Theory for Statistical Inference, Chapman and Hall, London, 1979.

25. R. Pyke, The supremum and infimum of the Poisson process, Ann. Math. Statist. 30 (1959), 568-576.

26. V. Reiner and G. M. Ziegler, Coxeter-associahedra, Mathematika 41 (1994), 364-393.

27. H. Robbins, A one-sided confidence interval for an unknown distribution function, Ann. Math. Statist. 25 (1954), 409 
28. H. Ruben, On the evaluation of Steck's determinant for rectangle probabilities of uniform order statistics, Comm. Statist. A-Theory Methods 5 (1976), 535-543.

29. R. Schneider, Convex Bodies: The Brunn-Minkowski Theory, Encyclopedia of Mathematics and Its Applications, Vol. 44, Cambridge University Press, Cambridge, 1993.

30. G. R. Shorack and J. A. Wellner, Empirical Processes with Applications to Statistics, Wiley, New York, 1986.

31. R. P. Stanley, Two poset polytopes, Discrete Comput. Geom. 1 (1986), 9-23.

32. R. P. Stanley, Enumerative Combinatorics, Vol 1, Wadsworth \& Brooks/Cole, Monterey, CA, 1986. Second printing, Cambridge University Press, New York, 1997.

33. R. P. Stanley, Parking functions and noncrossing partitions, Electron. J. Combin. 4 (1997), R20.

34. R. P. Stanley, Enumerative Combinatorics, Vol. 2, Cambridge University Press, New York, 1999.

35. J. D. Stasheff, Homotopy associativity of $H$-spaces. Trans. Amer. Math. Soc. 108 (1963), 275-292.

36. G. P. Steck, The Smirnov two sample tests as rank tests, Ann. Math. Statist. 40 (1969), 1449-1466.

37. G. P. Steck, Rectangle probabilities for uniform order statistics and the probability that the empirical distribution function lies between two distribution functions, Ann. Math. Statist. 42 (1971), 1-11.

38. L. Takács, Combinatorial Methods in the Theory of Stochastic Processes, Kreiger, Huntington, NY, 1977. 39. G. M. Ziegler, Lectures on Polytopes, Springer-Verlag, New York, 1995.

Received August 23, 2000, and in revised form June 4, 2001. Online publication April 30, 2002. 